\title{
SURVEY AND EVALUATION OF SECURE-DNS ALTERNATIVES THROUGH PASSIVE MEASUREMENTS
}

by

Ali Sadeghi Jahromi

A thesis submitted to the Faculty of Graduate and Postdoctoral Affairs in partial fulfillment of the requirements for the degree of

\section{Master of Computer Science}

Carleton University

School of Computer Science

(C) 2021

Ali Sadeghi Jahromi 
(C) Copyright by Ali Sadeghi Jahromi, 2021

All Rights Reserved 


\section{Abstract}

As security was not among the original DNS design goals, over ten secure-DNS schemes have been proposed to improve security and privacy during the name resolution process. One of the schemes is DNS-over-TLS (DoT), which relies on the Internet's PKI for establishing trust in recursive resolvers. The security research community has studied and improved security shortcomings in the web certificate ecosystem. DoT's certificates, on the other hand, have not been investigated comprehensively. This thesis analyzes the certificate ecosystem of DoT in comparison to HTTPS. The results are so far promising, as they show that DoT appears to have benefited from the PKI security advancements that were designed for HTTPS. The thesis then surveys secure-DNS schemes, and presents an evaluation framework to assess their security, availability, privacy, and anonymity benefits. Our evaluation illustrates that none of the DNS schemes secures the complete path of the domain name res-

olution. All rated schemes provide only partial security benefits in specific domain name resolution stages. The results shed light on the challenges of designing a comprehensive and widely-deployable secure-DNS scheme to secure the complete name resolution path. However, as some schemes can be combined, their resultant benefits can address individual shortcomings. 


\section{ACKNOWLEDGMENT}

I would like to thank the following people, without whom I would not have been able to complete this research:

First and foremost, my supervisor, Dr. AbdelRahman Abdou, for his support and valuable feedback on my research. He was a great mentor and guided me on the right path in my thesis journey.

Thanks to my examination committee members Dr. Paul Van Oorschot and Dr. GuyVincent Jourdan, for their careful review of my thesis and helpful comments that improved my work. I would also like to thank the CISL/CCSL members for their valuable feedback on my research during our meetings. Additionally, I am also thankful to the MADWeb anonymous reviewers for their feedback on my paper.

Last but not least, I would like to thank my parents for their love and support throughout my life. Without them, I would not have the courage and strength to chase my dreams. 


\section{TABLE OF CONTENTS}

Page

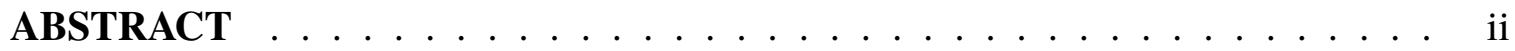

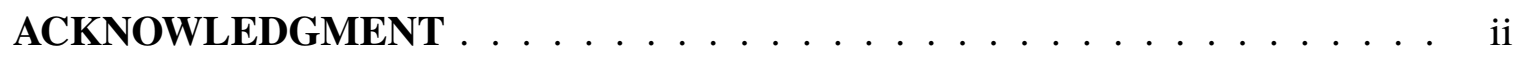

LIST OF TABLES $\ldots \ldots \ldots \ldots \ldots \ldots \ldots \ldots \ldots$ vii

LIST OF FIGURES $\ldots \ldots \ldots \ldots \ldots \ldots \ldots \ldots \ldots$ viii

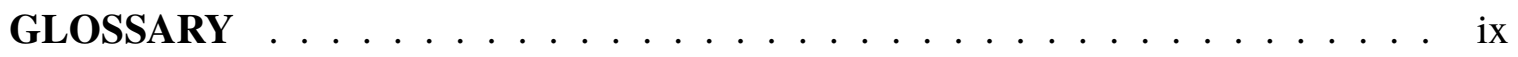

\section{CHAPTER}

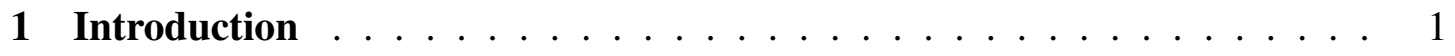

1.1 Motivation . . . . . . . . . . . . . . . 3

1.2 Problem Statement . . . . . . . . . . . . . . . . . . . 4

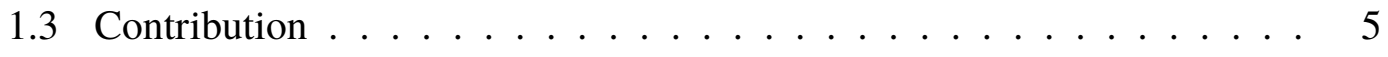

1.3.1 Thesis Outline $\ldots \ldots \ldots \ldots \ldots \ldots \ldots$

2 Background and Related Work . . . . . . . . . . . . . 7

2.1 Background and Context $\ldots \ldots \ldots \ldots \ldots \ldots$

2.1.1 DNS Working Procedure $\ldots \ldots \ldots \ldots$

2.1 .2 DNS Resolution Stages $\ldots \ldots \ldots$ 
2.1.3 EDNS Client Subnet (ECS) . . . . . . . . . . . . . . . . . 9

2.1.4 DNS Privacy Extensions . . . . . . . . . . . . 10

2.1.5 Security Deficits . . . . . . . . . . . . . 11

2.2 Related Work . . . . . . . . . . . . . . . . . . . . . . 12

3 DoT Certificate Ecosystem . . . . . . . . . . . . . . . . . . . 15

3.1 DoT and HTTPS Certificate Ecosystems _ . . . . . . . . . . . 15

3.2 Data Collection and Analysis Methodology . . . . . . . . . . . 17

3.2.1 Ethical Considerations . . . . . . . . . . . . . . . . . 18

3.3 Certificate Issuers $\ldots \ldots \ldots$

3.4 Certificate Parameters and Characteristics _. . . . . . . . 21

3.4 .1 Issuer-not-Found . . . . . . . . . . . . . . . . . . . . 22

3.4.2 Self-Signed Certificates . . . . . . . . . . . . . . . 23

3.4.3 Expired Certificates . . . . . . . . . . . . . . . . . . . 24

3.4 .4 Expiry Windows . . . . . . . . . . . . . . 25

3.4.5 Public-key and Hash Function . . . . . . . . . . . . . . . . 27

3.5 Inclusion in Certificate Transparency Logs . . . . . . . . . . . . . 28

3.6 Concluding Remarks . . . . . . . . . . . . . . . . . 31

4 Comparative Evaluation of Secure-DNS Alternatives . . . . . . . . . . 32

4.1 DNS Security and Availability Properties . . . . . . . . . . . . 33

4.1 .1 Security Properties . . . . . . . . . . . . . . . 33

4.1 .2 Availability Properties . . . . . . . . . . . . . . 37

4.2 Privacy and Anonymity Properties _ . . . . . . . . . . . . . 39

4.3 Threat Models . . . . . . . . . . . . . . . . . . . . . 42

4.3 .1 Assumptions . . . . . . . . . . . . . . . . . . 42

4.3.2 Compromising Privacy Versus Anonymity . . . . . . . . . . . 43

4.3 .3 Threats . . . . . . . . . . . . . . . . . . . 43

4.4 Schemes Evaluation . . . . . . . . . . . . . . . . . . 46

4.5 Rating Vanilla DNS . . . . . . . . . . . . . . . . . . . . 46

4.6 A Survey of Secure-DNS Alternatives _ . . . . . . . . . . . 47

4.6 .1 DNSSEC . . . . . . . . . . . . . . . . . . 47

4.6 .2 DNSCurve . . . . . . . . . . . . . . . . . . . . 49

4.6 .3 DNSCrypt*V3: . . . . . . . . . . . . . . 51

4.6.4 DNS-over-TLS (DoT) . . . . . . . . . . . . . . . . 53

4.6.5 DNS-over-HTTPS $(\mathrm{DoH}) \ldots \ldots \ldots \ldots$ 
4.6.6 DNS-over-QUIC (DoQ) . . . . . . . . . . . . . . . 56

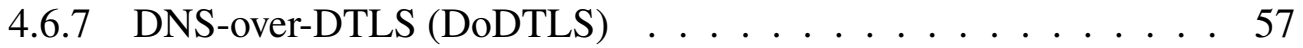

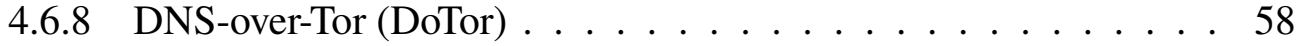

4.6 .9 Oblivious DNS (ODNS) . . . . . . . . . . . . . . 60

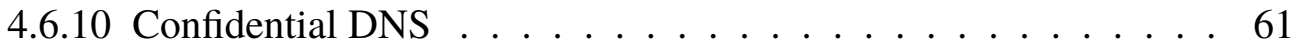

4.7 Insights from the Table . . . . . . . . . . . . . . . 64

4.7.1 Association Among Properties _. . . . . . . . . . . . 64

4.7 .2 Comparison of Schemes . . . . . . . . . . . . . . . 66

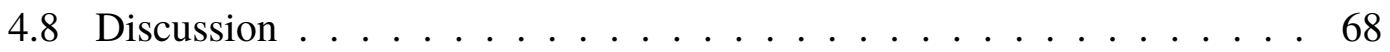

5 Conclusion and Future Work . . . . . . . . . . . . . . . . . 72

5.1 Future Work . . . . . . . . . . . . . . . . . 73

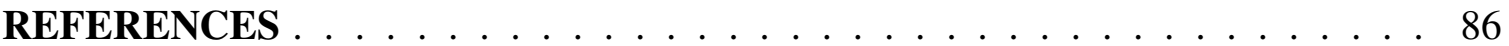




\section{LIST OF TABLES}

3.1 Subject fields containing IP addresses, and their count among self-signed and non-self-signed certificates. . . . . . . . . . . . . . . . . 24

3.2 Distribution of public and private IP addresses. . . . . . . . . . . . . 24

3.3 Main hash functions . . . . . . . . . . . . . . . . . 28

4.1 Comparative evaluation framework of secure-DNS alternatives . . . . . . 63 


\section{LIST OF FIGURES}

2.1 Complete DNS resolution path, broken into two stages: Pre-recursive-resolver (Stage-1) and Post-recursive-resolver (Stage-2). . . . . . . . . . . . . . . 9

2.2 QName minimization: recursive resolver only sends the necessary part of client's query to the ANSes. 'A': IPv4 address record query, 'NS': Name Server record query . . . . . . . . . . . . . . . . 11

3.1 Distribution of certificate issuers and subjects in DoT and HTTPS. . . . . . 19

3.2 The proportion of certificates issued by the 5-most popular CAs in DoT, and the proportion of HTTPS certificates issued by these CAs. . . . . . . . . . . 20

3.3 Proportion of valid and invalid certificates in our datasets, and the reasons of invalidity. . . . . . . . . . . . . . . . 22

3.4 Distribution of expiry windows in DoT and HTTPS certificates. White bars represent HTTPS, gray DoT. The proportion issued by each of the two most popular issuers (namely, GlobalSign and Let's Encrypt) is illustrated. . . . . 26

3.5 Asymmetric key algorithms (length) in DoT and HTTPS certificates. . . . . 29

3.6 Embedded SCTs in DoT and HTTPS certificates. The distance between red marked points with $\mathrm{a}, \mathrm{b}$, and the black points marked with $\mathrm{c}$, $\mathrm{d}$ are representing the portion of certificates in DoT and HTTPS that were logged to the Digicert CT-2 log. . . . . . . . . . . . . . . . . . 30 
4.1 DNS entities that can be targets for attacks (blue), and the type of attacks on these $($ red). . . . . . . . . . . . . . . . . . . . . 45

4.2 Vanilla-DNS: provides none of the properties in the framework. . . . . . 46

4.3 DNSSEC: Designed to provide end-to-end integrity and data origin authen-

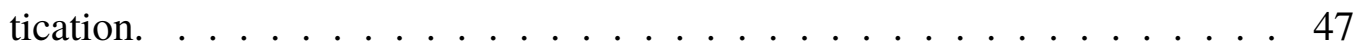

4.4 DNSCurve: Only adds security properties to the communication between the resolver and ANSes. . . . . . . . . . . . . . . . . . . . . . 49

4.5 DNSCrypt*V3: Provides security properties in Stage-1, plus using relay server to enhance clients' privacy and anonymity. . . . . . . . . . . . 51

4.6 DoT: Sending DNS queries over TLS in Stage-1. . . . . . . . . . . 53

4.7 DoH: Sending DNS queries over HTTPS using URIs and HTTP methods 55

4.8 DoQ: Sending DNS using QUIC transport protocol. . . . . . . . . . . . 56

4.9 DoDTLS: Sending DNS using DTLS transport protocol. . . . . . . . . 57

4.10 DNS-over-Tor: Based on Cloudflare's hidden resolver structure [89]. . . . . 58

4.11 Oblivious DNS: Adding confidentiality and Privacy to the DNS queries [91]. 60

4.12 Confidential-DNS: Adding confidentiality in both stages. . . . . . . . . . 62 


\section{Glossary}

ANS Authoritative Name Server

AS Autonomous System

CDN Content Delivery Network

CT-log Certificate Transparency log

DNS Domain Name System

DNSSEC Domain Name System Security Extensions

DoDTLS DNS-over-DTLS

DoE DNS-over-Encryption

DoH DNS-over-HTTPs

DoQ DNS-over-QUIC

Dot DNS-over-TLS

DoTor DNS-over-Tor

DRDoS Distributed Reflection Denial of Service

DS Delegation Signer 
EDNS Extension Mechanisms for DNS

HTTP Hypertext Transfer protocol

IMAP Internet Message Access Protocol

ISP Internet Service Provider

KSK Key-Signing-Key

MitM Man In The Middle

ODNS Oblivious DNS

ODoH Oblivious DNS-over-HTTPS

PKI Public Key Infrastructure

RR Resource Record

RRSIG Resource Record Digital Signature

SCT Signed Certificate Timestamp

SNI Server Name Indication

TLD Top Level Domain

TLS Transport Layer Security

ZSK Zone-Signing-Key 


\section{Chapter 1}

\section{Introduction}

In 1987 Domain Name System (DNS) was proposed and standardized as a distributed hierarchical name database of the Internet resources [70,69]. DNS is a critical component of the Internet; every communication that uses a domain name starts with a name resolution to discover the associated IP address. Humans, IoT devices, and applications use domain names to communicate with their intended services over the Internet. Like other primary Internet protocols (e.g., HTTP, IMAP), at the dawn of the Internet, DNS was designed and implemented without security [70,69]. Governments block or redirect DNS messages to suit their political agenda. For example, censorship in different countries such as Iran or India takes place through Internet Service Providers (ISPs) and intermediate devices that perform DNS hijacking/manipulation to redirect clients to arbitrary, or black hole, destinations $[11,95,74,44,73]$. As the DNS is a rich source of information, MORECOWBELL and QUANTOMDNS are projects deployed by the NSA, which were used to monitor web and DNS traffic around the world and hijack DNS traffic [42]. Therefore, large-scale DNS monitoring and manipulation have been negatively influencing privacy and security of DNS clients worldwide.

The proliferation of numerous IoT devices exacerbates these problems; 50 billion IoT devices are expected to be Internet-connected by 2025 [52]. Such devices resolve domain 
names to communicate with their cloud backends. The lack of privacy in DNS enables intermediate entities, such as ISPs and other Autonomous Systems (ASes), and also recursive resolvers to identify the type of IoT devices that a user owns [7]. Besides, due to the lack of security, DNS is vulnerable to active attacks, such as Kaminsky's DNS cache poisoning [56], DNS hijacking, DNS Data manipulation [80], amplification reflection attacks [5], and the use of DNS to leverage attacks in other areas [41, 55, 45].

In order to secure DNS and mitigate the existing attack vectors, different solutions such as transmitting DNS messages over secure sessions (e.g., Transport Layer Security (TLS) [49]), opportunistic encryption of DNS messages (e.g., Confidential DNS [103]), or adding integrity and data origin authentication to the DNS messages (e.g., DNSSEC [8, 10, 9]) were proposed. These schemes typically secure specific sections of DNS, which leaves other attack vectors in other sections. Additionally, some DNS schemes rely on specific infrastructures such as the Internet PKI in DoT [49], or root trust anchor and chain of trust in DNSSEC, which adds additional deployability complexity and new attack vectors to the DNS ecosystem. Besides, there often is a trade-off in secure-DNS schemes between the security properties they provide, and the overhead and complexity they add to the domain name resolution procedure. Such a trade-off should be considered in their evaluation.

In this thesis, firstly, we comparatively analyze the security of the DoT and HTTPS certificate ecosystems to observe how promising the security of X.509 certificates is in DoT. Secondly, considering the fundamental differences in the name resolution procedure of various DNS schemes, we present a systematization of knowledge into DNS security alternatives by (1) surveying the literature of secure DNS, commenting on the threat model they were designed to address, (2) creating an evaluation framework to investigate the degree by which DNS schemes secure the DNS messages in the domain name resolution. 


\subsection{Motivation}

DoT relies on the Internet PKI for authentication and managing X.509 certificates [49, 18]. Previous certificate ecosystem attacks, such as DigiNotar in 2011, affected the privacy and security of $\sim 300,000$ Iranian Gmail clients [62]. Therefore, improper implementation of DoT, such as using weak parameters in certificates or not appending certificates to CT-logs, also leads to eavesdropping, Man in the Middle (MitM), and pharming attacks, which harms the security and privacy of DoT clients. Unlike HTTPS, whose certificate ecosystem has been investigated thoroughly in the past decade $[36,24,1,98]$, there is no detailed study on the DoT certificate ecosystem. As part of this thesis, we investigate the security of the DoT certificate ecosystem.

Focusing on secure-DNS schemes, one realizes that each typically provides security in a specific part of the domain name resolution procedure. The partial absence of security in the name resolution path leads to privacy and security issues, and there is no complete overview of the security benefits that these schemes provide. We design an evaluation framework to measure the security of DNS schemes in the whole path of the name resolution.

Additionally, there are different threat agents in the DNS ecosystem. Governments can be interested to access DNS contents to eavesdrop and censor the web. Companies such as Mozilla, Comcast, or Google have been advocating for secure recursive-resolvers, as part of their commitment to customers' privacy. However, using DoT and DoH does not hide clientrelated information from these centralized resolvers, and they can build models of browsing behavior based on the clients' behaviors for their business-related purposes. Security-aware clients want to preserve the privacy of their DNS queries because they can reveal personal information about them. We are not aware of a security and privacy evaluation framework that comprehensively captures the benefits and limitations of the various schemes in the DNS ecosystem. Developing this framework would help future DNS designs to consider different design goals. 


\subsection{Problem Statement}

DoT is a relatively new DNS scheme, which has been deployed by large public DNS resolvers (e.g., Google, ${ }^{1}$ Cloudflare, and Comcast [32]). Previous research [63] showed that the number of DoT clients was increasing during their research period. Transferring DNS messages over secure TLS sessions adds new TLS-related vulnerabilities to the DNS ecosystem. Therefore, the security of DoT relies on the PKI entities and the issued certificates. HTTPS is another protocol that sends requests and responses over secure-TLS sessions. As HTTPS precedes DoT by over fifteen years, its security has much evolved. The first problem is that the security of DoT certificate ecosystem has not been investigated comprehensively. In Chapter 3, we comparatively analyze DoT and HTTPS certificate ecosystems and PKI parameters to analyze how secure the certificate ecosystem is in DoT. Such an evaluation can reveal whether DoT certificates exhibit strong security parameters. Weak security, such as weak cryptographic keys (problem: compromised key), self-signed certificates (problem: lack of revocation measures), and not including certificates in CT-logs (problem: malicious or mistakenly-issued certificates) are investigated in this thesis.

Due to the long-standing lack of security in DNS, many alternatives have been proposed to enhance its security and privacy. Such alternatives have benefits and drawbacks in different facets (e.g., security, availability, privacy, and anonymity). Due to the lack of an objective analysis of such alternatives, there is no clear overview of their differences and similarities. In Chapter 4, firstly, we survey secure-DNS schemes to explain their advantages, disadvantages, and working procedures. Secondly, we define objective security, availability, privacy, and anonymity evaluation parameters to assess secure-DNS schemes. Finally, we rate the surveyed schemes using our evaluation parameters. Since all such alternatives provide partial security to the DNS ecosystem, in Chapter 4 we also discuss potential solutions to complement their security benefits. Hence, the second problem is the lack of a

\footnotetext{
${ }^{1}$ https://developers.google.com/speed/public-dns/docs/dns-over-tls
} 
framework to assess properties that secure-DNS schemes provide.

\subsection{Contribution}

This thesis makes two contributions: investigating security of DoT certificate ecosystem, and designing an evaluation framework to evaluate the security benefits of secure-DNS alternatives. Details follow.

Contribution-1: We follow a passive measurements approach, using two certificate datasets from Rapid7, ${ }^{2}$ gathered on ports 443 (HTTPS) and 853 (DoT) to evaluate the DoT certificate ecosystem in comparison with HTTPS. Comparing these two ecosystems would shed light on the security of DoT certificates. This work was published in NDSS MADWeb workshop 2021 [88]. The thesis supervisor, Prof. Abdou, is a co-author of this workshop paper, and has thus contributed parts of the writing.

Contribution-2: In order to evaluate the security of various DNS schemes, we define objective security properties based on primitive security goals (e.g., confidentiality, integrity, and availability) and DNS-related vulnerabilities (e.g., censorship and privacy leaks). Then we examine secure-DNS alternatives based on our defined framework to assess the security benefits that they provide in the complete domain name resolution path.

\subsubsection{Thesis Outline}

The rest of this thesis is organized as follows:

- Chapter 2: Provides a brief background on DNS, and explores related work on DNS vulnerabilities and secure-DNS schemes.

- Chapter 3 (Contribution-1): Investigates security of the DoT certificate ecosystem in comparison to HTTPS.

\footnotetext{
${ }^{2}$ https://opendata.rapid7.com/
} 
- Chapter 4 (Contribution-2): Surveys secure-DNS alternatives, and presents a comparative evaluation framework to measure security, availability, privacy and anonymity of DNS alternatives.

- Chapter 5: Concludes the thesis, and discusses future work. 


\section{Chapter 2}

\section{Background and Related Work}

\subsection{Background and Context}

In this section, we give background on DNS-related techniques used to provide security, privacy and increase efficiency.

\subsubsection{DNS Working Procedure}

DNS was established in 1987, and standardized in RFCs-1034 [70], 1035 [69]. The primary goal was to design a consistent, scalable, and decentralized system to translate domain names into IP addresses. No security goals were set at that time [70]. Throughout this thesis, we refer to the original (insecure) DNS system as Vanilla DNS.

As Figure 2.1 illustrates, a typical name resolution procedure in Vanilla DNS starts with a client-side stub-resolver sending a query including QName to a recursive resolver. QName is the specified domain name in a DNS query that a client wants to resolve (e.g., carleton.ca), and stub-resolvers are the client-side DNS components that transmit DNS queries to the recursive resolvers [70]. This query (Step 1) is known as recursive query. In recursive queries, resolvers either return the final answer to a DNS query, or an error. A recursive resolver traverses the domain name hierarchy, sending iterative queries to the Authoritative 
Name Servers (ANSes). In iterative queries, if the ANSes do not have an answer to a query, they return the best answer that they have, which is the address of another ANS lower in the DNS hierarchy. The recursive resolver sends iterative queries to the ANSes until it gets the answer to the client's query, or an error occurs. Iterative queries are queries in Step 2 till $n-2$. Finally, in Step $n$, the recursive resolver responds to the client with an answer or an error if it fails to resolve that domain name.

\subsubsection{DNS Resolution Stages}

The name resolution process can be broken down into two stages.

Stage-1: Stage-1 is the communication path between the client (stub-resolver) and the recursive resolver (Figure 2.1). Based on the configured recursive-resolver on the clientside, the intermediate entities in Stage-1 will vary. The client can use a recursive-resolver inside its local network, or that of the ISP. The recursive resolver's address is typically configured automatically by the DHCP protocol if the client does not specify one manually. There are also public resolvers over the Internet, which are typically run and maintained by trusted (i.e., by their users) commercial companies (e.g., Google, Cloudflare). Callejo et al. [22] in 2019 found that $13 \%$ of DNS clients world-wide have been using public recursive resolvers. Moura et al. [71] shows that $30 \%$ of the DNS queries to their studied '.nz' and '.nl' country code Top Level Domains (ccTLDs) are generated by the recursive resolvers, which resided in 5 top cloud providers. Lu et al. [63] also found an increasing pattern in using DoT and DoH with public resolvers. As such, public recursive resolvers, which are neither in the clients' local networks nor their ISPs, form a significant portion of DNS queries over the Internet. ASes are a set of interconnected prefixes (networks) having a single unambiguous routing policy under control of one or multiple operators [43]. An ISP would have the first AS that transfers all of the client's Intenet traffic. Therefore, unlike other ASes in Stage-1 that are likely to differ based on the remote addresses that the client communicates, the ISP 


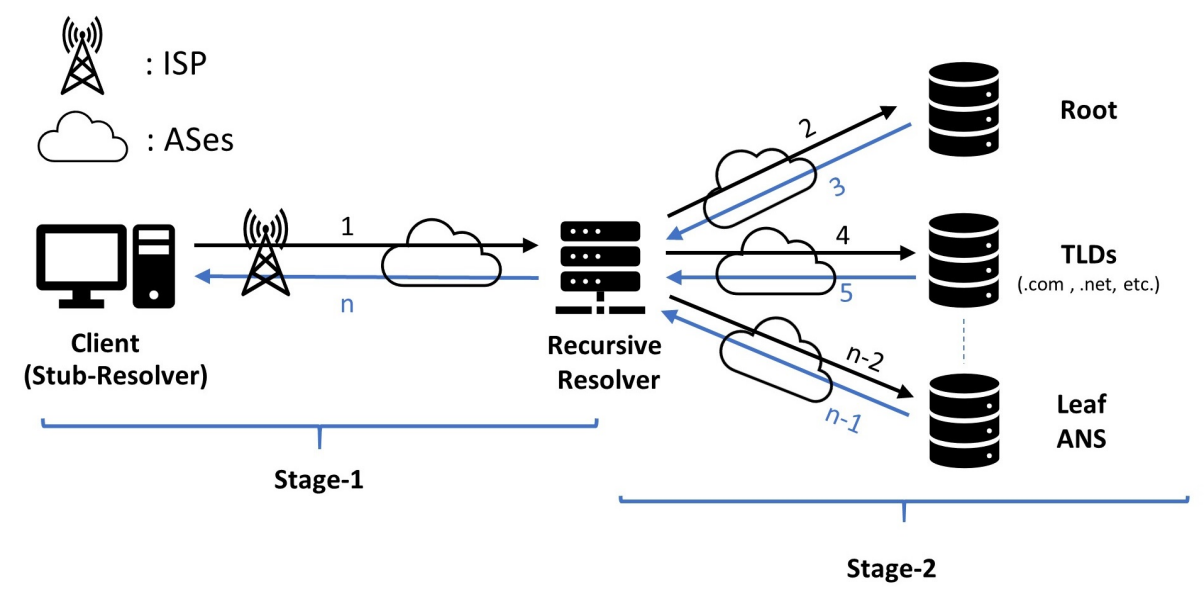

Figure 2.1 Complete DNS resolution path, broken into two stages: Pre-recursiveresolver (Stage-1) and Post-recursive-resolver (Stage-2).

is the fixed entity that transfers all of the client's Internet traffic.

Stage-2: When the client's query reaches the recursive resolver and the Recursion Desired (i.e., RD bit is set): if the recursive resolver does not have a cached answer to the query, Stage- 2 communications between the recursive resolver and ANSes begins. In Stage-2, ANSes do not usually perform recursion themselves. If an ANS does not have the response to the client's DNS query, it will respond with the address of the next DNS server, which is in a lower position in the hierarchy, which might be authoritative for the queried domain name. This procedure continues until the recursive resolver finds the ANS for the queried domain name, or fails. As Figure 2.1 depicts, Stage-2 of the DNS resolution path starts from Step 2 to Step $n-1$. Steps $n-2$ and $n-1$ are the query and response from the ANS. Stage-2 queries and responses are typically non-confidential, and transferred through different ASes over the Internet. Moura et al. [71] have shown that the communication between cloud-based resolvers and ANSes uses mostly UDP, with a small portion of TCP.

\subsubsection{EDNS Client Subnet (ECS)}

ANSes can only see the recursive resolver's IP address; they do not see client's IP address. Content Delivery Networks (CDNs) aim to minimize latency by connecting clients to the 
geographically closest servers. A CDN's ANS thus requires the client's IP address to return the nearest surrogate server's IP address. ECS is a DNS extension to indicate the client's IP address or subnet inside the DNS queries headed to the ANSes. It uses the extension mechanisms for DNS 'EDNS(0)' [26]. Although it is suggested to truncate IPv4 and IPv6 to 24 and 56 bits respectively to protect clients' privacy, this is not implemented as part of the protocol, and clients can send their 32/128 bit IPv4/v6 addresses alongside their queries [26].

If the client includes ECS in the queries, when the ECS-supporting recursive resolvers receive a DNS query, they will forward the ECS as part of their queries to all ANSes in the DNS hierarchy. Therefore, all ANSes from the root and Top Level Domains (TLDs), down to the name server, which is authoritative for the domain name, can view the ECS in the resolver's queries. As a result, the client's IP address (and consequently its geographic location) will be disclosed to the queried ANSes. Anonymization efforts, including Tor, proxy servers, or relay servers, are thus rendered futile. Despite improving performance in CDN-based services, ECS decreases clients' privacy—more on this can be found in Ch. 4.

\subsubsection{DNS Privacy Extensions}

EDNS(0) Padding: This is a padding option provided on top of the DNS extension mechanisms, which allows the clients and servers to add an arbitrary number of octets to their queries and responses to mitigate size-based correlation attacks [68]. EDNS(0) padding thus provides privacy for the encrypted DNS messages. However, Siby et al. [93] found that adding EDNS(0) padding solely will not prevent traffic analysis attacks, and adversaries would still be able to access DNS content using ML-based analysis techniques. QName minimization: Recursive resolvers typically send the full QName (i.e., client's queried domain name) to the ANSes. Therefore, ANSes can gather information about the queried domain names from resolvers, independent of whether ECS (Sec. 2.1.3) is included in those 


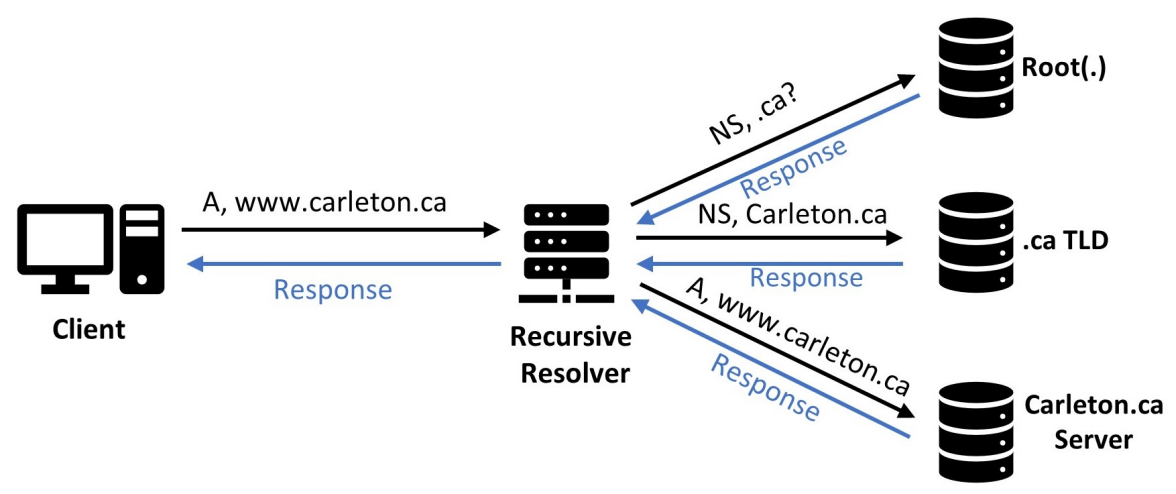

Figure 2.2 QName minimization: recursive resolver only sends the necessary part of client's query to the ANSes. 'A': IPv4 address record query, 'NS': Name Server record query

queries. As a result, the privacy of the clients will decrease by disclosing full QNames, and ANSes can gain information about the clients that use these resolvers. QName minimization was proposed to minimize the included QName in the resolver queries (RFC-7816 [20]). Therefore, as the resolver traverses the DNS hierarchy to resolve a domain name, it includes only the necessary part of the QName in the queries.

Figure 2.2 shows an example of QName minimization. When the recursive resolver resolves $w w w . c a r l e t o n . c a$, it will only sends .ca to the root server instead of the complete QName.

\subsubsection{Security Deficits}

DNS does not implement primary computer security goals such as confidentiality, integrity, data origin authentication, and entity authentication, which makes it vulnerable to attacks such as DNS cache poisoning [96], DNS flooding [65], DNS hijacking [96], DNS Distributed Reflection Denial of Service (DRDoS) [108], manipulation [80], and eavesdropping [19]. The threat space targets different entities in the name resolution path, and securing the whole name resolution path to mitigate attacks requires securing multiple different paths between different entities. 


\subsection{Related Work}

As DNS has been used for over 30 years, extensive literature studied DNS-related vulnerabilities and attacks from different aspects. Pearce et al. [80] demonstrates that recursive resolvers in specific countries (e.g., Iran or China) actively manipulate over $20 \%$ of DNS queries. Zou et al. [110] categorized DNS attacks into five groups, and provided defense mechanisms based on existing DNS schemes. In another comprehensive survey, Khormali et al. [58] studied DNS threats and vulnerabilities, DNS research methods, and the impact of DNS entities' (i.e., clients, resolvers, name servers, and hosting providers) security on the DNS ecosystem. Torabi et al. [101] investigated the systems designed to passively monitor and analyze (e.g., using ML models) DNS traffic to detect DNS-related threats (e.g., Fastflux domains, Malicious domains). They also proposed their own DNS monitoring system that detects malicious DNS-related behaviors at a near real-time speed.

Regarding threats that name server delegations can cause in the domain name system, Ramasubramanian et al. [82] showed that domain names over the Internet usually depend on over 40 authoritative servers in the path of their name resolution. Thus, vulnerable, dependent servers could be used as leverage for DNS hijacking attacks by redirecting the resolvers to their arbitrary malicious name servers.

In 2005, DNS Security Extensions (DNSSEC) documents were published by Arends et al. $[8,10,9]$ that added authenticated denial of existence, integrity, and data origin authentication to the DNS responses based on cryptographic signatures implemented in authoritative name servers. DNSSEC was thus designed to mitigate DNS cache poisoning and DNS manipulation attacks. However, DNS queries and responses are still transmitted in plain-text-middle entities (e.g., ISPs and ASes) could eavesdrop on DNS packets. The extra fields, namely digital signatures and the Extension mechanism fields (EDNS), led to DNS amplification and DRDoS attacks. In DNSSEC, amplification reflection attacks can multiply the amount of DoS traffic up to 44 times, as a result of the extra fields in the DNS 
responses [5]. Additionally, DNS packets are still susceptible to censorship by the middle entities in the name resolution procedure. Dai et al. [28] studied DNSSEC's public key parameters and its chain of trust, and found that $\sim 20 \%$ of the Second Level Domains (SLDs) could not establish a successful chain of trust to the root name server. Chung et al. [25] show that only $12 \%$ of DNSSEC-enabled resolvers validate DNSSEC signatures, and $31 \%$ of DNSSEC-enabled name servers provide DNSSEC records. Therefore, DNSSEC suffers from complex key management and deployment errors [27, 25]. In general, as of this writing, DNSSEC remains undeployed widely, neither by resolvers nor authoritative name servers.

Like other Internet protocols (e.g., HTTP and SMTP) that were secured by TLS, in 2015, the idea of transferring DNS messages over TLS began to gain wide attention [109]. (DoT) was then standardized [49] in 2016 to protect DNS messages between the stuband recursive- resolver from manipulation and eavesdropping [49, 19]. DNS-over-HTTPS (DoH) [46] was then proposed in 2018 [49, 46]. DoT uses port 853 and sends DNS queries over secure TLS sessions [49]. In comparison, DoH uses port 443, which is the same as other HTTPS traffic, and sends DNS queries over HTTP requests through TLS sessions [46]. From security perspective, they are almost the same as each other, as they work over TLS. Lu et al. [63] briefly evaluated DNS-over-Encryption (DoE) protocols- the general class of protocols designed to secure the stub-to-recursive resolver communication, including DoT and DoH. They [63] provided a multi-aspect comparative assessment of DoE protocols, and evaluated security of each aspect using two parameters, namely "Uses standard TLS" and "Resists DNS traffic analysis". They showed that during their research period, the number of clients that adopted DoT and DoH, using public resolvers (e.g., Google and Cloudflare) had increased [63].

Recently, due to emerging client privacy concerns and increasing centralization of DNS with large public resolvers, research efforts are dedicated to new protocols and mechanisms to decouple a client's QName from its IP address, protecting the client's privacy. DNS-over- 
Tor [89], ODNS [91], and DNSCrypt*V3 [30] are examples of privacy-preserving alternatives with different approaches. We explain them in detail in Chapter 4. 


\section{Chapter 3}

\section{DoT Certificate Ecosystem}

DoT was proposed to secure the stub-to-recursive resolver stage (Stage-1) of the domain name resolution procedure [49]. Facebook and Cloudflare designed a pilot project to experiment with DoT in Stage-2 (i.e., recursive-to-ANS) of the name resolution procedure. ${ }^{1}$ However, due to the increased CPU usage, the amount of transmitted traffic, and latency, ANSes are unlikely to be willing to deploy DoT in Stage-2. DoT relies on the Internet's PKI, and uses X.509 certificates to associate public keys to DoT resolvers.

In this chapter, we compare DoT's certificates with HTTPS'. HTTPS has been in use long before DoT, and its security has since evolved. As DoT is a relatively new protocol, assessing its certificate ecosystem illustrates how much security improvements it has inherited from HTTPS.

\subsection{DoT and HTTPS Certificate Ecosystems}

We investigate whether DoT would be susceptible to classic (or historic) PKI shortcomings, such as invalid/self-signed certificates, weak cryptographic parameters, or fraudulent certificates issued by compromised non-existent CAs. Over time, browsers enhanced HTTPS

\footnotetext{
${ }^{1}$ https://engineering.fb.com/2018/12/21/security/dns-over-tls/
} 
security by stringent certificate validation and indispensable demand of security features, like the placement of certificates in Certificate Transparency (CT) logs [97]; CAs have accordingly been stepping-up their issuance standards. It is unclear how many of the browserimplemented reinforcements for HTTPS are adopted in DoT, and how the relatively lax security in DoT affects issued certificates. For example, successful authentication [3] and encryption are unnecessary in DoT depending on the client's configured usage profile. If a client software is configured to use opportunistic usage profile [49], analogous to opportunistic encryption, the client only enforces security properties when possible. If not possible, it resolves the DNS query without encryption and authentication. In the strict mode [49] when the TLS certificate gets processed by the stub-resolver, name resolution requires successful authentication and encryption to resolve a domain name.

We present results upon comparing a random sample of DoT and HTTPS certificates collected from Rapid7 [84]. Particularly, this chapter contributes results upon comparing DoT and HTTPS certificates for the following aspects:

- Distribution and characteristics of certificate issuers (Sec. 3.3).

- Certificate parameters, including validity windows and cipher-suites (Sec. 3.4).

- Proportion and distribution of certificates in CT-logs (Sec. 3.5).

Our results highlight non-major differences between both ecosystems, including differences in: the dominant $\mathrm{CA}$, certificate validity, and cryptographic properties. The proportion of invalid certificates appears almost similar in both ecosystems, likewise the expiry windows and cryptographic functions. We also found almost equivalent rates of CT-log inclusion in both ecosystems. These results suggest that so far, the deployment and usage of DoT certificates in practice are comparable to HTTPS, and are not significantly affected by the lack of strict security checks in stub-resolvers. 


\subsection{Data Collection and Analysis Methodology}

In Stage-1, DoT clients verify identity of a recursive resolver using X.509 certificates. This authentication in DoT is unilateral-the recursive resolver authenticates itself to the client. As DoT proposed to secure DNS traffic in Stage-1, the certificates studied in this research are gathered from the recursive resolvers over the Internet. We employ a passive measurement methodology, where we used two certificate datasets gathered by Rapid7's Project Sonar ${ }^{2}$ in March 2020: one for HTTPS, the other DoT. Project Sonar actively scans the Internet for different services, similar to Censys [35]. Project sonar scans for a specific service port over the IPv4 address space using Zmap [84]. Further information and protocol metadata are then extracted by communicating with the IP addresses over the open ports, analogous to banner grabs [35]. The gathered data then gets released on the Open Data web page [84]. A possible inaccuracy in scanning based on IP addresses are the IP addresses that host multiple similar services, and their certificates are distinguished using Server Name Indication (SNI). In such cases, the scan returns only one of the certificates unless the client specifies the SNI in the "Client Hello" message.

The DoT and HTTPS datasets that we used were gathered by Rapid7 on the $4^{\text {th }}$ and $23^{\text {rd }}$ of March 2020, respectively. They consist of X.509 certificates and their fingerprints collected from scanning DoT resolvers (port 853) and HTTPS webservers (port 443), respectively [84]. The DoT dataset consists of $\sim 10 \mathrm{~K}$ certificates, the HTTPS $\sim 9.3 \mathrm{M}$. However, $\sim 54 \%$ of the DoT certificates (collected over ports 853 ) were also found in the HTTP certificate dataset (collected over port 443). The overlapping certificates belonged to less than a quarter of the DoT certificate owners (942 of 4632 unique subjects); these owners apparently chose to use the same certificate for both DoT and HTTPS.

We used the OpenSSL toolkit, SCTcheck, and the Pyopenss l library to parse and process certificates in both datasets. For certificate validity, we used OpenSSL's verify utility.

\footnotetext{
${ }^{2}$ https://www.rapid7.com/research/project-sonar/
} 
Validating a certificate also involves chain validation [18]. For that, we relied on Mozilla's intermediate CA certificate lists ${ }^{3}$ released on April 2020, ${ }^{4}$ and the certificate root store of Ubuntu 18.04. OpenSSL's verify utility stops validating certificates when the first invalidity reason is encountered. For example, if the certificate is self-signed and expired, the tool would report only one of those two invalidity reasons and quit. As such, for every certificate in our dataset, we had to write our own scripts to list all reasons of invalidity.

Finally, we noticed that many certificates had an IP address placed as a string in the Subject and Issuer fields. Those were mostly self-signed certificates. We used MaxMind's IP/Country database to geolocate these addresses.

\subsubsection{Ethical Considerations}

We categorize ethical considerations related to the explained passive measurement into two parts. The first relates to considerations that Rapid7 takes into account in their active scans. Rapid7 provides an opt-out email address to exclude IP addresses whose administrators are unwilling to participate in the scans. Rapid7 also states that their scans will not harm the targeted entities in the IPv4 address space. The second part of ethical considerations is on the individuals or organizations using Rapid7's datasets. Rapid7's Terms of Service (ToS) dictates that users must not: "Do anything illegal or in violation of the rights of others, including unlawful access or damage to computers, and facilitate or encourage illegal activity" [84]. We followed the ToS, and as we performed passive investigations on the datasets, there were no additional ethical requirements to consider.

\footnotetext{
${ }^{3}$ https://wiki.mozilla.org/CA/Intermediate_Certificates

${ }^{4}$ The difference between the scanning time and the intermediate certificates release time might lead to a negligible inaccuracy as Mozilla releases the list of intermediate certificates daily.
} 


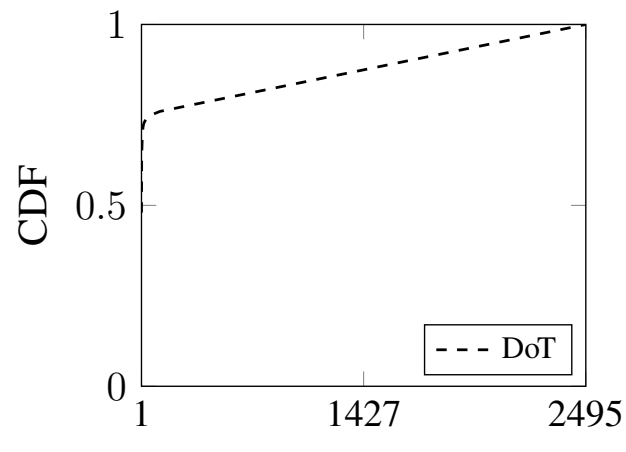

Certificate Issuers

(a)

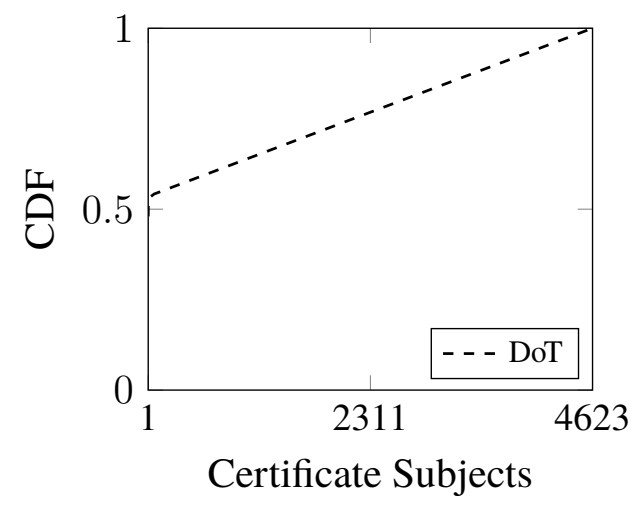

(c)

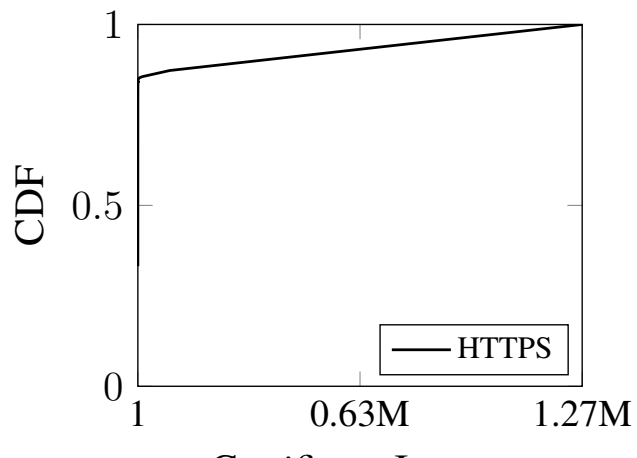

Certificate Issuers

(b)

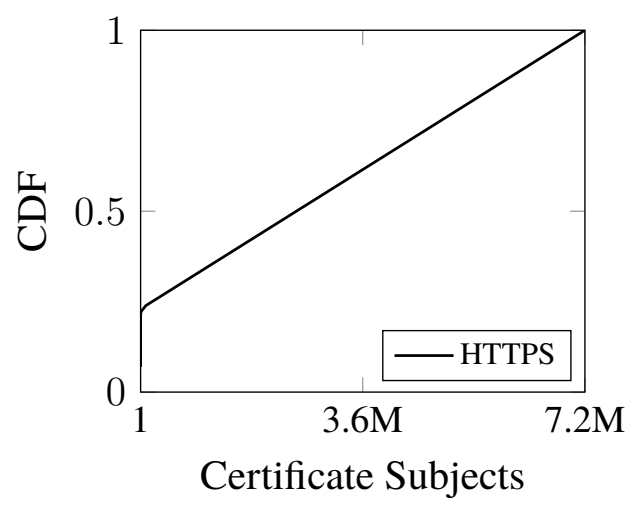

(d)

Figure 3.1 Distribution of certificate issuers and subjects in DoT and HTTPS.

\subsection{Certificate Issuers}

In this section, we investigate the distribution of certificate issuers and subjects among DoT and HTTPS certificates.

Figures 3.1a and 3.1b show CDFs of certificate issuers. For DoT, 105 of 2495 issuers were responsible for $\sim 76 \%$ of DoT certificates. In HTTPS, $\sim 91 \mathrm{~K}$ of $\sim 1.27 \mathrm{M}$ issuers were responsible for $87 \%$ of HTTPS certificates. Although many issuers were untrusted, about two-thirds of the certificates in both ecosystems were issued by trusted issuers. The long tails in Figures 3.1a and 3.1b represent issuers that issued very few certificates. The majority of those ( $>90 \%$ for both ecosystems) were either non-existent issuers or untrusted CAs, and they mostly issued one certificate. Collectively, these have issued $<37 \%$ of certificates in 
both ecosystems.

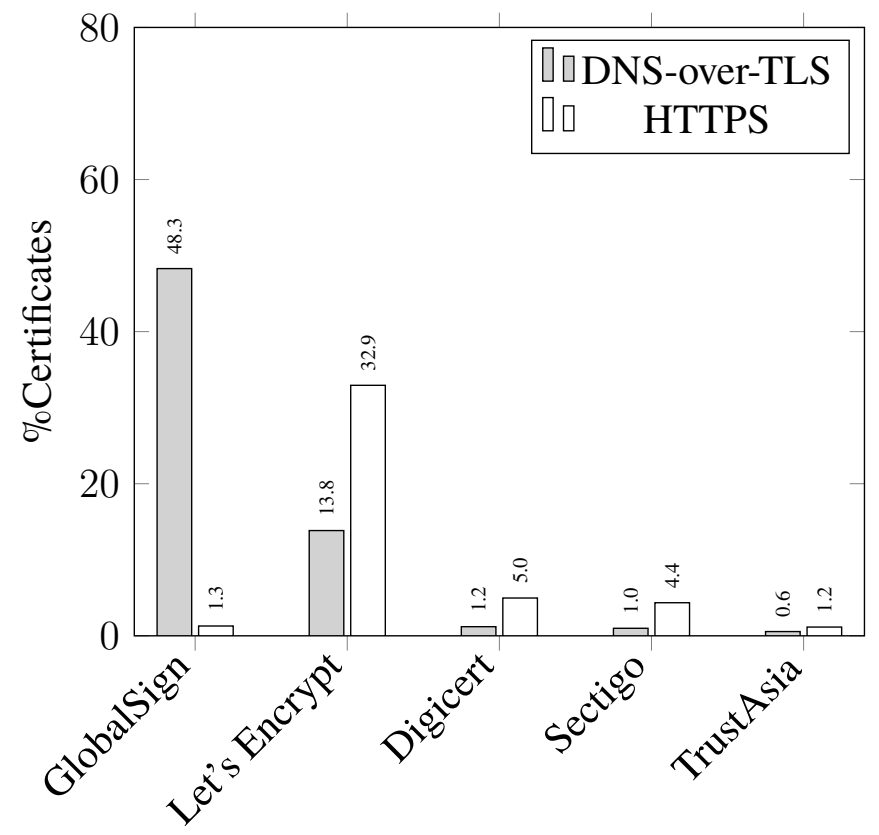

Figure 3.2 The proportion of certificates issued by the 5-most popular CAs in DoT, and the proportion of HTTPS certificates issued by these CAs.

Figures 3.1c and 3.1d show a CDF of the Subject field, which identifies the certificate owner (the entity that owns the public key). Many ( $\sim 50 \%)$ DoT certificates in our dataset are owned by a single entity. This organization is incapsula.com. Imperva Incapsula is an American cybersecurity company, which provides cloud-based security solutions to its customers. We assume that as a part of their security solutions, they deployed these DoT resolvers for their clients. The distribution was less skewed in HTTPS, with Technicolor network devices owning $~ 7 \%$ of HTTPS certificates in our dataset.

Figure 3.2 shows the top five DoT issuers and the proportion of HTTPS certificates that they have issued. GlobalSign is the dominant CA in DoT. However, the majority of the certificates GlobalSign has issued belong to a single owner (incapsula.com), rather than being distributed amongst many DoT services. The $\sim 10 \mathrm{~K}$ DoT certificates in our dataset belong to $\sim 4.6 \mathrm{~K}$ unique owners; the numerous DoT certificates that GlobalSign CA has issued belong to only 31 of the $4.6 \mathrm{~K}$ owners. In contrast, the $13.8 \%$ of DoT certificates issued by 
Let's Encrypt belong to 1348 owners. So Let's Encrypt can be considered equally popular in the DoT ecosystem, despite issuing significantly fewer DoT certificates (13.8\%). For HTTPS, Let's Encrypt remains the dominant certificate issuer, which aligns with previous literature [90, 1], whereas GlobalSign has only issued $1.3 \%$ of the HTTPS certificates in our dataset.

Summary. GlobalSign and Let's Encrypt are the two most popular CAs in the DoT ecosystem. The former was founded in $1996,{ }^{5}$ and the latter has established a strong security reputation worldwide. Both CAs employ the Automatic Certificate Management Environment (ACME) protocol [13], which automates certificate renewal. Together they are responsible for almost two-thirds of the DoT certificates in our dataset. Our analysis on certificate issuers, thus, shows no outstanding signs of security concerns in the DoT certificate ecosystem regarding issuers.

\subsection{Certificate Parameters and Characteristics}

We now move to certificate parameters, specifically focusing on the characteristics of invalid certificates (Sec. 3.4.3 to Sec. 3.4.1), expiry windows (Sec. 3.4.4), and cryptographic properties (Sec. 3.4.5).

Figure 3.3 shows the proportion of valid and invalid certificates in both ecosystems, with invalid certificates clustered by reasons of invalidity. As a certificate can be invalid for multiple reasons (e.g., expired, and has an untrusted issuer), the sum of the proportions exceeds $100 \%$ on the vertical axis. As shown, $65 \%$ of the DoT certificates in our dataset were valid, compared to $58 \%$ for HTTPS. We now analyze and compare the top three invalidity reasons across both ecosystems.

\footnotetext{
${ }^{5}$ https://web.archive.org/web/20010205010600/http://globalsign.net/company/index.cfm
} 


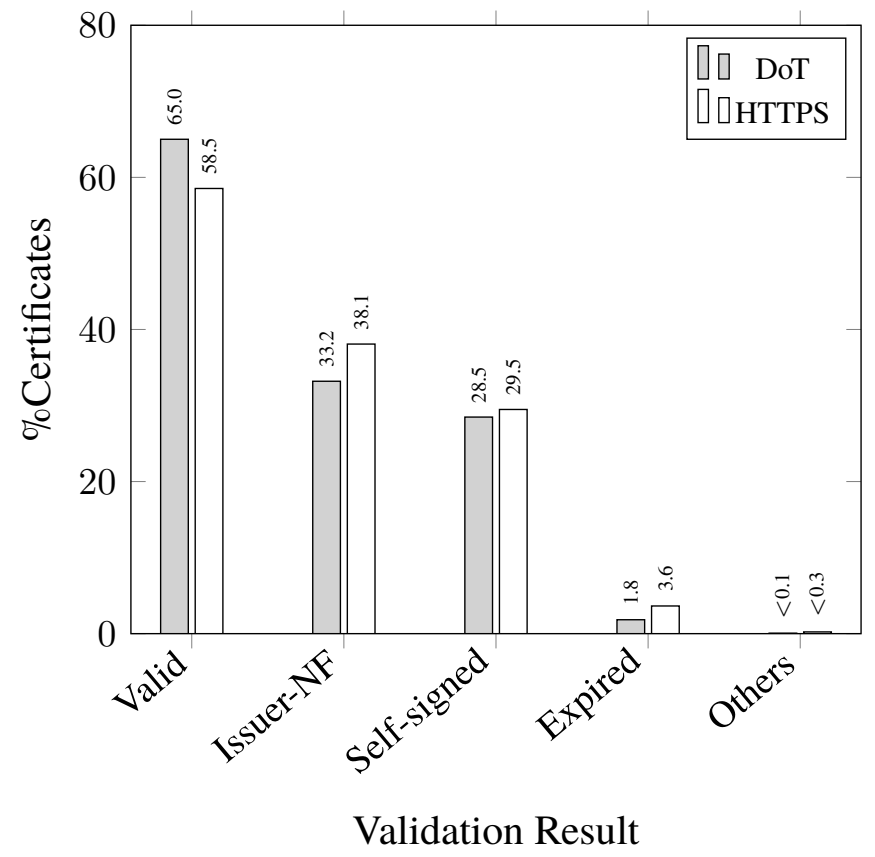

Figure 3.3 Proportion of valid and invalid certificates in our datasets, and the reasons of invalidity.

\subsubsection{Issuer-not-Found}

The most common invalidity reason is Issuer Not Found "Issuer-NF", meaning the leaf certificate issuer could not be found in the list of trusted (intermediate) issuers or root certificates. The majority of the DoT issuer-NF certificates were issued by Fortinet network devices, which also constitute a considerable proportion of issuer-NF certificates in our HTTPS certificate dataset. But the most popular issuer of issuer-NF HTTPS certificates was Technicolor. These certificates had long expiry-windows ( $\geq 5$ years), which raises further concerns about their security. Chung et al. [24] explained that many client-side network devices regularly generate invalid certificates for secure remote administration, which increase the proportion of invalid certificates in an ecosystem. Overall, issuer-NF certificates in both ecosystems were mostly issued by network devices rather than CAs, and had relatively long expiry windows. 


\subsubsection{Self-Signed Certificates}

A self-signed certificate is one where the Issuer field is the same as the Subject. The entity in the PKI that guarantees a public key belongs to a recursive resolver is the CA. With selfsigned certificates, there is no guarantee that the public key is associated with the specified domain name in the certificate. Additionally, CAs are responsible for revoking certificates e.g., by including the vulnerable certificates in Certificate Revocation Lists (CRLs) [18]. Using self-signed certificates is harmful in TLS based ecosystems. For example, lack of enough entropy in generating public key pair may lead to weak private keys, or if the private key gets compromised, revoking or rotating the certificate is not as quick and precise as using a CA issued certificate [17]. In the DoT ecosystem, clients (e.g., IoT devices or Internet users) have to trust a self-signed certificate without any guaranteed evidence. Although self-signed certificates mitigate passive attacks, they expose users to active attacks, such as resolver impersonation, MitM, and pharming attacks.

From Figure 3.3, the proportion of self-signed certificates in our data set was almost equal in both ecosystems: $28.5 \%$ of DoT certificates and $29.5 \%$ of HTTPS. There were $\sim 2.4 \mathrm{~K}$ unique issuers responsible for issuing the $2.85 \mathrm{~K}$ self-signed DoT certificates (the 28.5\% in Fig. 3.3) - no significant dominant of self-signed certificates in DoT. The two most popular CN strings in self-signed DoT certificates were "Server" and "localhost," respectively, constituting $8 \%$ and $5 \%$ of the self-signed DoT certificates. In contrast, $\sim 0.9 \mathrm{M}$ unique issuers have issued the 2.7M self-signed HTTPS certificates (29.5\% in Fig. 3.3), suggesting that the distribution of issuers among self-signed HTTPS certificates is more skewed than in DoT. The most popular two self-signed HTTPS certificate issuers were "Vigor Router" (5\%) and "192.168.1.1" (4\%).

Of the self-signed certificates, 61\% (DoT) and 15\% (HTTPS) had a string that mimics an IP address structure in the CN fields. Table 3.1 shows that using IPv4 addresses as the subject field is only present in self-signed certificates. From Table 3.2, public IP ad- 
dresses form $>80 \%$, and $>99 \%$ of the found IP addresses in HTTPS and DoT self-signed certificates, respectively. Upon geolocating the public IP addresses, we found that DoT's addresses map to 17 countries and HTTPS' to 201, which expectedly illustrates the size and distribution difference between both ecosystems. Half the HTTPS self-signed certificates with an IP address in the CN fields are located in the DoT's 17 countries. Therefore, despite minor differences between DoT and HTTPS self-signed certificates in our dataset, their proportion, distribution, and characteristics generally appear comparable.

Table 3.1 Subject fields containing IP addresses, and their count among self-signed and non-self-signed certificates.

\begin{tabular}{l|lcc}
\hline Data Set & Subject Field & Self-Signed & Not Self-Signed \\
\hline \multirow{2}{*}{ DoT } & IP address & 1,736 & 0 \\
& Non IP address & 1,100 & 7,119 \\
\hline \multirow{2}{*}{ HTTPS } & IP address & 404,231 & 0 \\
& Non IP address & $2,344,244$ & $6,573,467$ \\
\hline
\end{tabular}

Table 3.2 Distribution of public and private IP addresses.

\begin{tabular}{lcc}
\hline IP address & HTTPS $(\%)$ & DoT $(\%)$ \\
\hline Public & $324,571(80.4)$ & $1,724(99.3)$ \\
Private & $79,160(19.6)$ & $12(0.7)$ \\
\hline
\end{tabular}

\subsubsection{Expired Certificates}

A certificate has a Not-After field indicating the date and time when the certificate validity ends. We analyze the proportion of expired certificates in both datasets. We classify a 
certificate as expired if the Not-After field is before the time that Rapid7 has collected said certificates.

From Figure 3.3, although expired certificates in HTTPS is double that in DoT, they generally constitute a small proportion in both ecosystems. This can be attributed to CAs increasingly employing the ACME protocol. Investigating the top trusted CAs in both DoT and HTTPS, we observed from our dataset that all of these CAs except GoDaddy ${ }^{6}$ (as of this writing) are supporting ACME based certificate issuance and auto-renewal mechanisms to avoid unexpected expiration of certificates. ${ }^{7}$

We observe that the majority of expired certificates in both ecosystems are issued by Let's Encrypt: $\sim 78 \%$ for DoT, $\sim 60 \%$ for HTTPS. Despite being more popular among HTTPS certificates, the proportion of the expired certificates issued by Let's Encrypt in HTTPS is smaller compared to DoT. As such, there appears no positive correlation between Let's Encrypt-issued certificates and expired ones. Let's Encrypt enforces an expiry window of 90 days, which suggests that Let's Encrypt DoT certificates may not have been configured for auto-renewal. Next, we shed light on the certificate expiry windows in both ecosystems.

\subsubsection{Expiry Windows}

Shorter certificate lifespan is a healthier security practice [100], but might lead to functional and security problems as, e.g., management and operational costs increase [23]. Over the past several years, the security community has been advocating for shorter certificate expiry windows for the web. For example, before 2018, browsers required certificates to have an expiry window of 39 months or shorter, and later (from March 2018) agreed on accepting certificates with a maximum lifetime of 825 days ( $\sim 2$ years) [38, 79]. Google then announced the maximum accepted certificate lifespan by the Chrome browser would be 397

\footnotetext{
${ }^{6} \mathrm{https}: / /$ letsencrypt.org/docs/godaddy/

${ }^{7}$ GoDaddy provides independent mechanisms for automatic certificate renewal [39].
} 
days $(\sim 1$ year $) .{ }^{8}$ On September 2020 , Apple ${ }^{9}$ followed suit, accepting a maximum certificate life span of 398 days ( $\sim 1$ year).

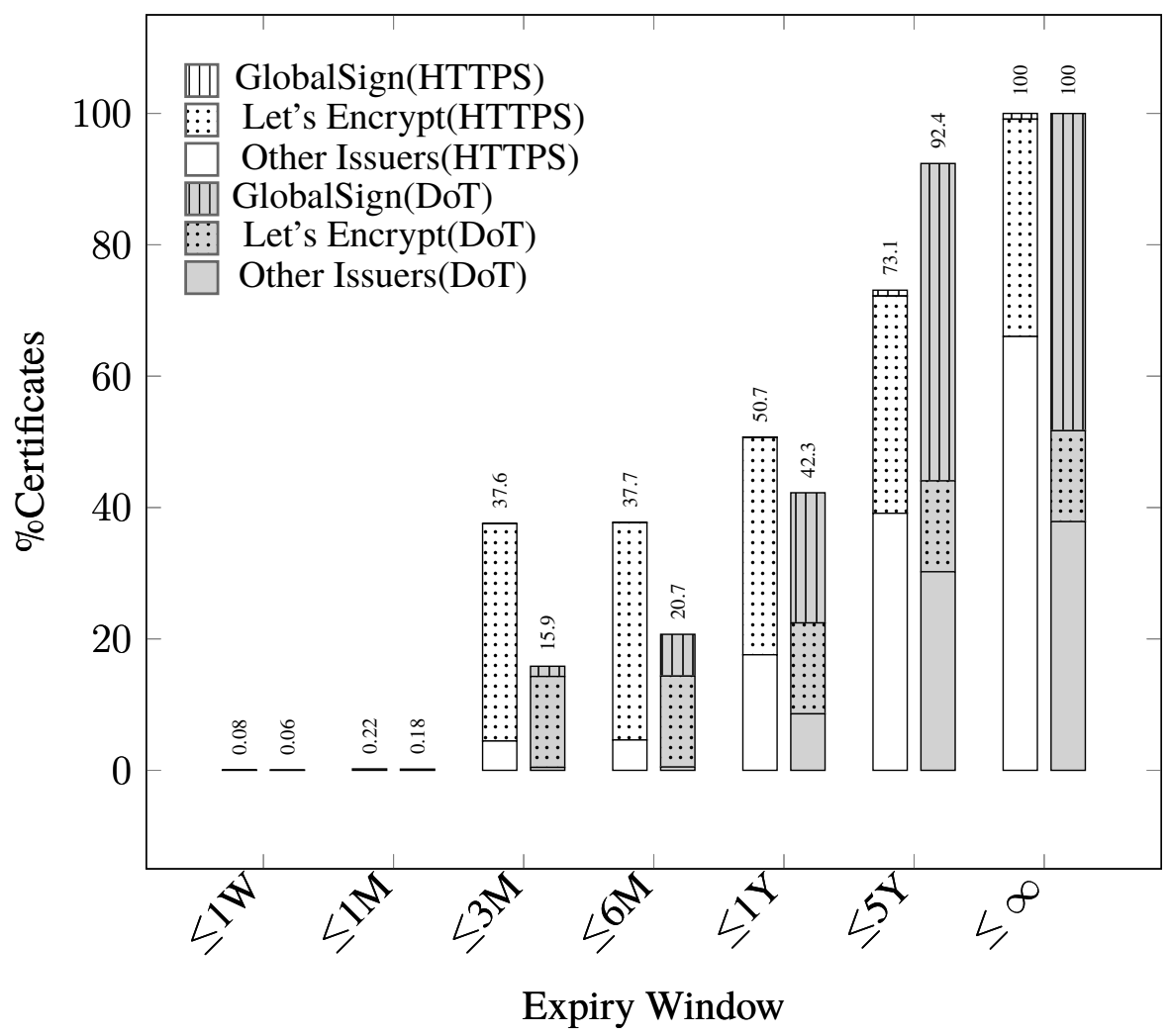

Figure 3.4 Distribution of expiry windows in DoT and HTTPS certificates. White bars represent HTTPS, gray DoT. The proportion issued by each of the two most popular issuers (namely, GlobalSign and Let's Encrypt) is illustrated.

Figure 3.4 shows a cumulative histogram of various expiry windows for the certificates in our dataset. A negligible number of certificates were issued with a lifespan of less than a month in both DoT and HTTPS certificates. Unexpectedly, a few number of (invalid) certificates had a negative expiry window-their not-after date was before their not-before. Very few certificates had less than one month expiry window. For HTTPS, 50\% of certificates in the $\leq 1 \mathrm{M}$ bin have been issued by trusted CAs, such as GTS, Sectigo, and Digicert. In contrast, all DoT certificates in that bin were issued by untrusted issuers. That is a minor plus point for HTTPS, but is potentially negligible due to the significantly small proportion of

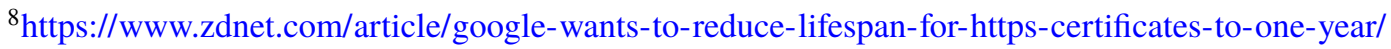

${ }^{9}$ https://support.apple.com/en-ca/HT211025
} 
certificates in that bin for both ecosystems. For the $\leq 3 \mathrm{M}$ bin, the HTTPS proportion is more than double the DoT, which can be attributed to the larger proportion of Let's Encrypt-issued certificates in HTTPS (see Sec. 3.3); the proportion of certificates the CA has issued in this bin is almost equal across both ecosystems— 88\% for HTTPS, $\sim 87 \%$ for DoT. Analogous patterns can be observed for the remaining bins.

The study of web certificates, before the foundation of Let's Encrypt CA, shows that trusted web certificates were mainly issued with one-year lifespan [102]. However, in our study, certificates with less than one-year lifespan are common in HTTPS, which can be attributed to (1) browsers (including Chrome and Safari) enforcing such shorter lifespans, and (2) the popular CA in HTTPS, Let's Encrypt, only issues certificates with 90 days lifespan. This appears to have not been carried over to the DoT ecosystem-over $70 \%$ of the certificates in DoT were issued with a lifespan of $\leq 2$ years by the top CAs, and Let's Encrypt has only issued $\sim 14 \%$ of DoT certificates in our dataset ( $c f$. Figure 3.2 ). A corollary of the above two points, although there is no short certificate lifespan enforcement in the DoT client-side tools, we observe that the majority of the DoT certificates were issued with relatively short expiry windows. The majority of certificates issued by the dominant DoT CA, Globalsign, had an expiry window between one and two years. Hence, DoT certificates are getting renewed in relatively short periods.

\subsubsection{Public-key and Hash Function}

In this section, we investigate public keys and hash function algorithms used in the DoT and HTTPS certificates as a part of their cryptographic properties. We rely on NIST recommendations for key management [12]. For asymmetric-key algorithms, $\leq 80$ bits, such as RSA-1024 and ECC (160-223), are considered weak. For hash functions, SHA-1 is also considered weak, and was formally deprecated in 2011 [12].

Figure 3.5 shows the asymmetric key algorithms used in both certificates in our datasets. 
RSA-2048, which has sufficient strength of 112 bits, is used by the majority of certificates in both ecosystems, with $\sim 87 \%$ in HTTPS and $~ 75 \%$ in DoT, which is similar to 2013 HTTPS public keys [36]. The weaker variant, RSA-1024, is five times more popular in DoT. The HTTPS ecosystem thus appears to have a slight advantage over DoT.

Table 3.3 Main hash functions

\begin{tabular}{lcc}
\hline Hash Algorithm & DoT & HTTPS \\
\hline SHA-256 & $\sim 98 \%$ & $\sim 96 \%$ \\
SHA-1 & $\sim 2 \%$ & $\sim 4 \%$ \\
\hline
\end{tabular}

Regarding hash functions, Table 3.3 shows that $>95 \%$ of certificates in both ecosystems used SHA-256, which has a security strength of 125 bits and is considered secure [12]. Only $2 \%$ of DoT certificates used SHA-1, but the proportion was double in HTTPS. Therefore, regarding the used hash functions, DoT certificates appear in a slightly better position. We note that there was a negligible proportion of $\leq 0.1 \%$ of other deprecated and nondeprecated hash functions, such as MD5 and SHA-384, in both ecosystems.

Summary. The characteristics of the DoT certificates appear to be comparable to HTTPS, especially in hash functions, certificate validity, and invalidity reasons. Minor exceptions exist in used public keys and expiry windows. However, similar to the previous section, we find no concerning evidence of lack of security in DoT certificates compared to the HTTPS.

\subsection{Inclusion in Certificate Transparency Logs}

CT-logs are append-only and publicly auditable databases of certificates that are designed to contain all of the issued TLS certificates [61]. Certificate issuers append their issued certificates to CT-logs. Upon the receipt of a certificate, CT-logs return a Signed Certificate Timestamp (SCT). The included certificate becomes publicly accessible, and the SCT is 


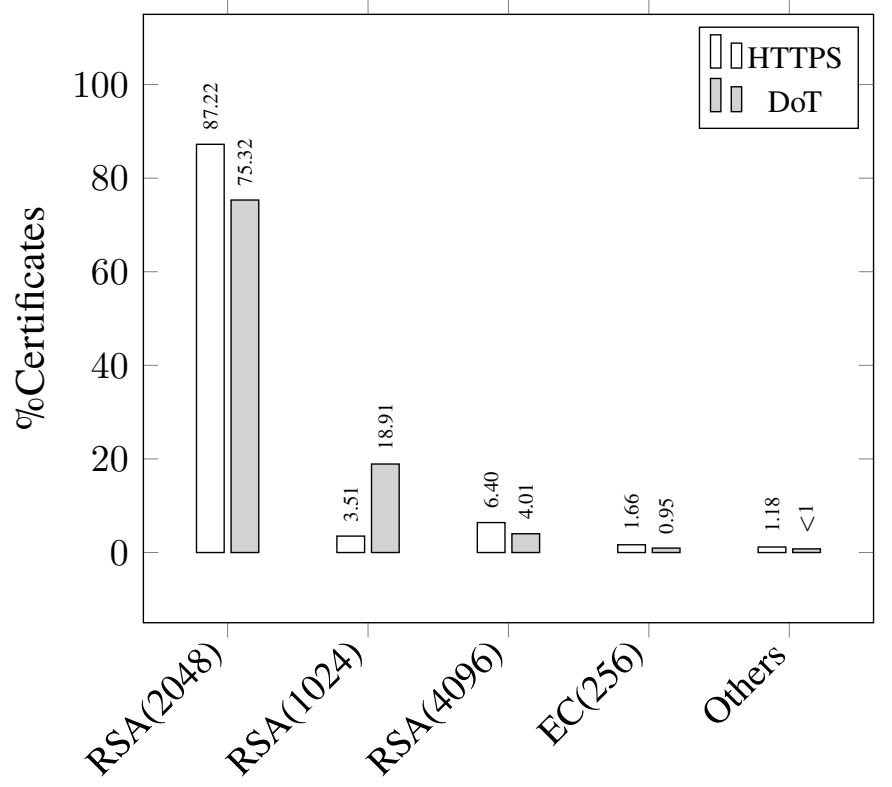

Key Algorithm(length)

Figure 3.5 Asymmetric key algorithms (length) in DoT and HTTPS certificates.

signed by the CT-log's private key to verify that the certificate has been included. Domain owners thus become aware of certificates issued for their domain, allowing them to detect maliciously or mistakenly issued certificates. We now compare two aspects: the proportion of certificates included in CT-logs, and the distribution of certificates among different CTlogs.

From our dataset, $\sim 61.4 \%$ and $\sim 66.8 \%$ of HTTPS and DoT certificates respectively had SCTs. Approximately $62 \%$ of the DoT certificates that contained SCTS were issued by either GlobalSign or Let's Encrypt. The top issuers adopted automatic inclusion of certificates in the CT-logs, and DoT certificates also benefited from this advancement.

Figure 3.6 shows a CDF of the distribution of certificates with SCTs among CT-logs. Both ecosystems exhibit a similar pattern. One notable point is that Digicert's CT2 log had been deactivated on May 2020 due to possible private key leakage [33]. In Figure 3.6, the line between points $a$ and $b$, and points $c$ and $d$ illustrates the proportion of DoT and HTTPS certificates logged into Digicert CT2 log. As the proportion in Digicert CT2 log is slightly 
greater in DoT, deactivation of this CT-log would negatively affect the DoT ecosystem more than HTTPS. Regarding popularity, Google Xenon2020 is the top CT-log among HTTPS certificates, whereas Sectigo Mammoth is the top among in DoT. Overall, the top three Google's CT-logs in HTTPS included more portion of certificates compared to the top three Google's CT-logs in DoT.

Summary. As browsers, like Chrome and Safari, ${ }^{10}$ enforced the inclusion of web certificates in CT-logs, CAs increasingly implemented CT-log inclusion policies [97]. Therefore, approximately all of the valid certificates in both ecosystems have been logged. The forced CT-log inclusion in the HTTPS ecosystem appears to have boosted the inclusion of DoT certificates.

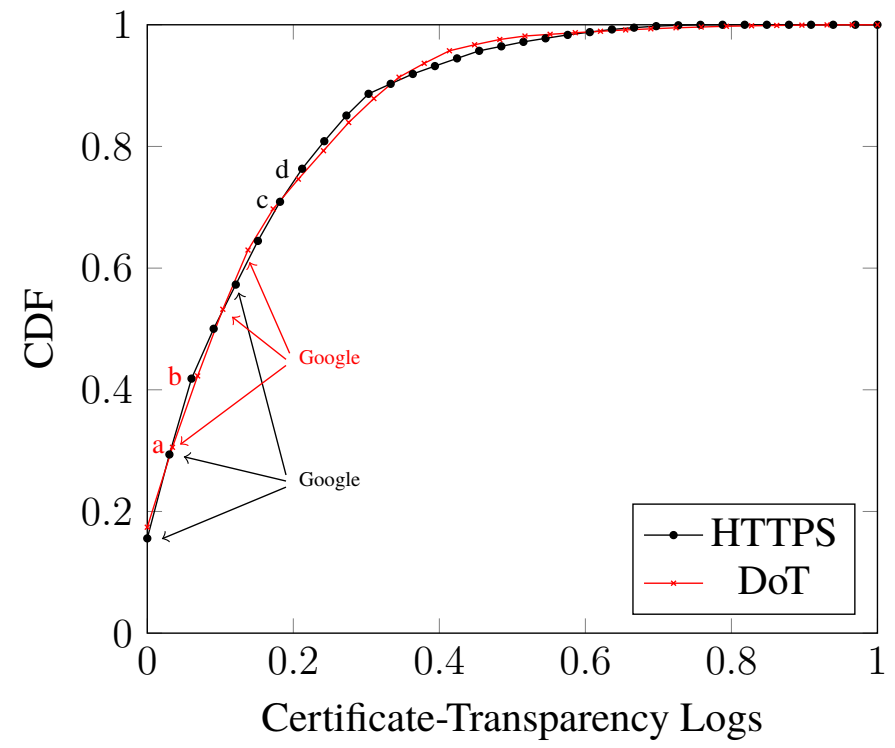

Figure 3.6 Embedded SCTs in DoT and HTTPS certificates. The distance between red marked points with $\mathrm{a}, \mathrm{b}$, and the black points marked with $\mathrm{c}, \mathrm{d}$ are representing the portion of certificates in DoT and HTTPS that were logged to the Digicert CT-2 $\log$.

\footnotetext{
${ }^{10}$ https://support.apple.com/en-ca/HT205280
} 


\subsection{Concluding Remarks}

The security of the HTTPS ecosystem has improved over time, with browser vendors, industry standards, CAs, and researchers coordinating efforts to advance aspects like CT-log inclusion, short certificate expiry windows, and improved cryptographic properties. Our analysis herein shows that the DoT ecosystem appears to have seized the momentum, reaping the benefits of the advancements made in HTTPS. We observed some differences between the invalid certificates of both ecosystems. Valid certificates, on the other hand, exhibit almost similar characteristics. Some certificates existed in both the DoT and HTTPS datasets. Those overlapping certificates are presumably stronger than ones present only in the DoT dataset because they (the overlapping) should satisfy the strict constraints of the HTTPS ecosystem. It would be unfair to judge the security of the DoT ecosystem with the DoT-only (non-overlapping) certificates because the overlapping ones are still used to establish DoT sessions in practice. As such, we took the conscious decision of conducting our analysis in both datasets as-is, without removing the overlapping certificates from the DoT dataset. A notable concern is that client-side DoT tools in the opportunistic mode can undermine the observed security strength of the DoT ecosystem. Client-side DoT tools must thus mimic the tenacity of modern browsers.

Now that we have analyzed some aspects of DoT in practice, we proceed to analyze the bigger picture of DNS schemes in the next chapter. 


\section{Chapter 4}

\section{Comparative Evaluation of Secure-DNS}

\section{Alternatives}

Many secure DNS schemes have been proposed in the literature. A subset of these have some degree of similarities in their operation. In this chapter, to clarify and evaluate the security properties that they provide, we first define nineteen DNS-related security, availability, privacy, and anonymity properties. We then survey secure-DNS schemes, discussing their security design goals and theory of operation. Finally, we assess each scheme based on our defined security properties to shed light on its strengths and weaknesses. We survey and evaluate ten different DNS schemes that have been proposed to enhance DNS security and privacy.

In summary, this chapter makes the following contributions:

- Defines a security, availability, privacy and anonymity evaluation framework for the DNS ecosystem (Sec. 4.1 and Sec. 4.2).

- Surveys secure-DNS schemes and techniques to improve security and privacy of the DNS protocol (Sec. 4.6).

- Evaluates the surveyed schemes using the evaluation framework (Sec. 4.7). 


\subsection{DNS Security and Availability Properties}

In this section, we define 12 security and availability and 7 privacy and anonymity properties that a secure DNS scheme can potentially provide. We realized such properties after performing extensive analysis to DNS threats and defences from academic literature and practice (e.g., data origin authentication or availability attacks $[87,59])$, or defined indirectly based on the previous literature (e.g., different types of censorship $[11,105])$.

\subsubsection{Security Properties}

S1 Resilient-to-Manipulation-in-Stage-1: In Stage-1, integrity is required for the messages between the client and the recursive resolver. Hash functions are typical means to achieve cryptographic integrity, and a small change to the DNS messages results in a new hash value. However, an active attacker can forge or manipulate DNS messages and recompute the new hash value. Hence, to mitigate active integrity attacks, message authentication in Stage-1 (S8) is required. DNS message integrity in both stages (see Sec. 2.1.2) must be assured to confirm that no unintentional, or simple manipulations took place during a domain name resolution.

Due to the lack of integrity in Vanilla DNS, unintentional and simple intentional changes occur to DNS messages. Consequently, authorized entities can not detect malicious or mistaken data modification by unauthorized parties. Integrity protection alone is insufficient to eliminate impersonation and manipulation attacks, and message authentication (S8) is required to verify message origins. DNS hijacking and DNS cache poisoning are two types of attacks that can occur in the absence of message authentication (see S8, S9) in the name resolution process. In Stage-1, these attacks redirect clients to arbitrary destinations. Resilient-to-Manipulation-in-Stage- 1 is achieved if a scheme provides integrity in Stage-1. 
S2 Resilient-to-Manipulation-in-Stage-2: Same as the previous property, except for Stage-2. To ensure the Resilient-to-Manipulation-in-Stage-2, a scheme has to provide integrity in Stage-2 between recursive resolver and the ANSes in the hierarchy of DNS. Cache poisoning attacks in Stage-2 target recursive resolvers and affect the clients that use a recursive resolver. This property is a necessary condition in Stage-2 to avoid recursive resolver cache poisoning. However, integrity is not sufficient, and data origin authentication (S9) is also required to mitigate cache poisoning against active attackers.

S3 Resilient-to-Exclusive-QName-Censorship: Large-scale DNS-based censorship occurs in different countries such as Iran [11], India [95, 106], and Pakistan [73]. These countries leverage DNS messages to exclusively censor specific QNames (see Sec. 2.1.1). To block a specific domain name, intermediate entities (e.g., ISPs) and network devices detect specific DNS queries based on their queried domain names and then redirect DNS queries to a fake local IP used as blackhole address $[11,106]$. The other method of censoring exclusive QNames is at the recursive resolver level when recursive resolvers respond with arbitrary black hole addresses to specific QNames. In order to prevent exclusive QName censorship, a DNS scheme has to provide confidentiality to hide QNames from the intermediate entities, integrity to prevent manipulation of DNS responses, and data origin authentication to prevent impersonation. Therefore, if a secure DNS scheme provides integrity, authenticity, and confidentiality of DNS messages, it is Resilient-to-Exclusive-QName-Censorship.

S4 Resilient-to-IP-based-Censorship: In IP-based censorship, the ISPs and routers inside a national network under control of a government can block destinations by IP addresses. For instance, as part of Tor censorship, Tor guard relays' and bridges' IP addresses are blocked by the firewalls of China [105]. Similarly, if a DNS scheme is limited to a number of resolvers that their IP addresses can be discovered easily, they are not Resilient-toIP-based-Censorship. For example, due to using a specific port number, DoT resolvers 
can be discovered over the Internet efficiently using Zmap [37], and then their IP addresses get blocked. If DNS was run over Tor, it can be censored by blocking the Tor guard relays' IP addresses.

S5 Resilient-to-Port-based-Censorship: A government can block a port number to censor a specific service. Therefore, secure-DNS schemes that use a distinguishable port number are susceptible to port-based censorship. On the other hand, schemes that disguise DNS traffic by using ports of other protocols, tend to provide this property. In that case, censoring traffic requires fingerprinting and traffic-analysis, which would be costly. For instance, DoH traffic uses port 443-its traffic gets merged with HTTPS; hence, censoring DoH would be costly (albeit not impossible).

S6 Resilient-to-Resolver-Replay-Attack: Beyond data origin authentication, entity authentication ensures that a specific entity is on the other end of a communication [67]. On top of entity authentication using freshness and liveness mechanisms ensures that a transmitted message is newly generated, mitigating replay attacks [67]. Freshness ensures that the message is generated recently and not replayed from previous queries and responses. Freshness is typically achieved by Time-Variant Parameters (TVPs), such as timestamps, that add uniqueness to the transmitted messages [77]. Liveness indicates that the communicating party is currently active [67]. Liveness can be achieved on top of freshness by defining time windows in which the TVPs are acceptable [67]. Most of the schemes do not mention means of liveness (e.g., using time windows) in their documentation. If a scheme provides means for freshness and entity authentication, we rate it as Resilient-to-Resolver-Replay-Attack.

Among the secure-DNS schemes, in the absence of freshness challenges (e.g., clockbased, sequence numbers, or nonce-based), replay attacks can occur. However, replay attacks in DNS are useful in a very specific attack vector. In the absence of entity authentication in Stage-1, the adversaries can replay the resolver's messages to direct the 
client to a destination after they exploited the vulnerabilities in the destination [57]. A DNS scheme that provides means for entity authentication and freshness mechanisms in Stage-1 is considered Resilient-to-Resolver-Replay-Attack.

S7 Resilient-to-ANS-Replay-Attack: Similar to Stage-1, in the absence of entity authentication and freshness mechanisms in Stage-2, the adversaries can replay DNS messages at any time after their generation. For instance, in DNSSEC, although the RRs are signed, they can be replayed by entities other than ANSes before the expiry of their signatures [107], and can thus be used to direct clients to exploited destinations [57]. If a scheme provides entity authentication and freshness mechanisms in Stage-2, it is Resilient-to-ANS-Replay-Attack.

S8 Resilient-to-Resolver-Impersonation: Data origin authentication (i.e., message authentication) is a stronger type of integrity. Data origin authentication guarantees that a specific entity is the source of the received messages, regardless of who sent the message or when it was sent [67]. This property is typically provided by Message Authentication Codes (MAC) that also ensure the integrity of the data [67]. The identity of the sender is usually verified by a trusted third party (e.g., CA in the Internet's PKI). Therefore, this property mitigates manipulation of DNS messages by the active attackers in Stage-1 between client and recursive resolver. A DNS scheme that mitigates resolver impersonation and DNS data manipulation attacks in Stage-1 through data origin authentication provides the property Resilient-to-Resolver-Impersonation.

S9 Resilient-to-ANS-Impersonation: Similar to the previous property (S8), data origin authentication provides Resilient-to-ANS-Impersonation. Lack of data origin authentication and integrity in Stage-2 leads to ANS impersonation and DNS cache poisoning attacks [56]. Therefore, lack of ANSes data origin authentication in Stage-2 allows an adversary to fill the cache of recursive resolvers with malicious records, which cannot get validated. This would be harmful to the DNS ecosystem, and affects all clients that 
rely on the victim recursive resolver.

\subsubsection{Availability Properties}

A1 Resilient-to-Resolver-DoS: In order to ensure the availability of the resources in a domain name resolution, recursive resolvers have to be accessible to legitimate users without being susceptible to DoS by malicious entities. However, perfect availability over the Internet is idealistic, as a sufficiently large volume of traffic can block any service. Nevertheless, some DNS schemes are more resilient to DoS attacks than others. The primary type of attack against availability is Distributed Denial of Service (DDoS), which overwhelm the resources of the target from multiple locations. The typical consumed resource is the target's bandwidth. Bandwidth depletion is a general type of DDoS that can target any Internet-connected service.

DDoS attacks on UDP-based DNS typically exhaust the CPU of the target [109]. Thus, CPU usage, response latency, and the number of unanswered queries increase significantly [109]. Additionally, due to the lack of enough information to detect spoofed packets in UDP-based DNS, rate-limiting based on source address is infeasible [109]. DDoS attacks on TCP-based application occupy all possible active connections so that legitimate users cannot have access to the service [109].

We define Resilient-to-Resolver-DoS based on the transport layer protocol that they use for the secure-DNS alternatives. In TCP-based DNS, SYN cookies can mitigate SYN flood attack [109]. Unlike UDP-based DNS, TCP-based DNS schemes can thus implement SYN cookies with rate-limiting technique to mitigate DDoS attacks. As the investigated schemes did not mention the use of SYN cookies and rate-limiting in their documentation, if a scheme works over TCP, as it has means to mitigate DDoS attacks, we consider it resilient against DDoS. A TCP-based DNS scheme in Stage-1 would thus be Resilient-to-Resolver-DoS. 
A2 Resilient-to-ANS-DoS: Similar to recursive resolvers, ANSes are also susceptible to DoS attacks, and we apply the same rule for rating resilience of ANSes to DoS: schemes where ANSes use TCP provide Resilient-to-ANS-DoS.

A3 Resilient-to-DRDoS: DNS responses are typically larger than queries. In Distributed Reflection Denial of Service (DRDoS) attacks, botnets take advantage of the connectionless UDP-based DNS infrastructure to amplify their attack and increase its effectiveness. For instance, the Mirai botnet in 2016 leveraged DNS infrastructure to amplify the amount of traffic that the zombies sent to targets $[6,2]$.

Similar to A1 and A2, TCP-based alternatives that implemented SYN cookies and ratelimiting mechanisms are resilient to DRDoS amplification attacks as they have the means to mitigate spoofed packets. Therefore, similar to A1 and A2, we assume that TCP-based alternatives can mitigate DRDoS attacks against resolvers and ANSes, while UDP-based alternatives are susceptible to DRDoS attacks.

In resolver or ANS DoS attacks, the adversaries flood the resolvers and ANSes. While in DRDoS attacks, target other Internet entities than the resolvers or ANSes. If a scheme has a 1 : 1 ratio for queries and responses (i.e., only reflection), the only advantage of DRDoS attacks is hiding the original source of attack from the target, and DRDoS attacks are not beneficial to the adversaries.

In our evaluation, we consider DRDoS with amplification-if a scheme protects resolvers and ANSes from DRDoS, by using TCP, or $1: 1$ response/query size ratio, it completely (O) provides Resilient-to-DRDoS. If a scheme secures one of the entities (resolves or ANSes), it is partially ( ) providing this property, and if it protects none of them, it is not Resilient-to-DRDoS. Simple reflection attacks (i.e., no amplification) are more general type of DRDoS that can be expanded into another column in the table. 


\subsection{Privacy and Anonymity Properties}

P1 Resists-ISP-Correlation-Analysis: ISPs reside in Stage-1 between a client and recursive resolver. Hence, an ISP can observe different types of traffic generated by the client. Regardless of confidentiality, if an ISP can distinguish DNS traffic from other transmitted traffic effortlessly, they can correlate the name resolution process to subsequent traffic coming from the client, and determine the resolved domain name.

A DNS scheme that mystifies DNS nature (P2) partially ( ) provides this property. For example, a scheme that applies traffic-flow security techniques [99], such as sending dummy traffic when not resolving a domain name, partially achieves Resists-ISPCorrelation-Analysis. Using non-specific port numbers also merges DNS traffic with other protocol's traffic and mitigates ISP correlation analysis.

In the schemes that conceal DNS nature (P3), the ISP is not able to detect that a name resolution is occurring. Therefore, these schemes completely (๑) provide Resists-ISPCorrelation-Analysis.

P2 Mystifies-Message-Nature-in-Stage-1: If DNS traffic is distinguishable in Stage-1, intermediate devices can block, redirect, or censor the encrypted DNS traffic and even force the client to fall back to Vanilla DNS. Besides, if the encrypted DNS is distinguishable, in some DNS alternatives (e.g., DoT), it is also susceptible to traffic analysis, which reveals the client's queried domain name [93]. When a scheme makes its traffic indistinguishable or makes the identification of DNS traffic from other traffic costly or complicated (i.e., not distinguishable by source/destination port/IP address or any other straightforward information), a scheme Mystifies-Message-Nature-in-Stage-1. For instance, some DNS schemes merge their traffic with other protocol's traffic, which mystifies their nature. Merging with other traffic increases the cost of separating DNS messages from the other traffic by intermediate entities in Stage-1. For instance, DNSCrypt 
uses port 443, which is the same as HTTPS, and merges its traffic with the web. Therefore, the ISP and the intermediate ASes cannot easily distinguish its traffic from the web, and distinguishing DNS traffic requires further traffic analysis. Another example is using traffic-flow security techniques [99], which does not allow the intermediate entities to figure out when an actual name resolution is occurring. Nevertheless, mystified DNS traffic can be detected by fingerprinting, destination-address, and traffic analysis methods, but traffic detection would be costly.

P3 Conceals-Message-Nature-in-Stage-1: Unlike P2, this property is beyond mystification. It is rewarded to schemes that anonymize the name resolution procedure by hiding the nature of DNS messages. For example, if the DNS messages are transmitted through Tor, the nature of DNS messages gets wrapped in Tor traffic cells-hidden in the Tor's network with layers of encryption. Therefore, although intermediate Stage-1 entities are able to identify Tor traffic, they cannot identify that a name resolution is occuring. Siby et al. [93] showed that DoH-over-Tor also resists ML-based traffic analysis that other protocols (e.g., DoT and DoH) fail to resist. Schemes that completely hide the nature of DNS messages in Stage-1 are represented with Conceals-Message-Nature-in-Stage1. This property is a subset of Mystifies-Message-Nature-in-Stage-1. That is, schemes that conceal the message nature also mystify such nature. Although there is no known research on traffic analysis of Vanilla-DNS-over-Tor, as the length of DNS messages is relatively small, traffic analysis attacks can still be possible to detect Vanilla DNS queries over Tor [21]. However, the ML-based traffic analysis methods would be costly and limited to the trained models.

P4 Hides-Client-Identity-from-Resolver: Although confidentiality limits the access of unauthorized parties to DNS message content, authorized parties (e.g., recursive resolvers) typically have access to the clients' name resolution information, such as the domain name that the client requested (QName) and the client's IP address. In Stage-1, this in- 
formation could be gathered by public resolvers and later used to analyze clients' browsing behavior. Bird et al. [16] showed that browsing history can uniquely identify users in different periods. Furthermore, in the case of Internet of Things (IoT) devices, name resolution can reveal the IoT device type to the entities who have access to the DNS messages, such as the recursive resolvers or intermediate ASes [7]. Some DNS schemes use techniques such as proxies, relay servers, or Tor network to enhance the privacy of the client against the recursive resolvers, hiding the client's IP address. Undoubtedly, if a client adds ECS in the DNS queries, the anonymizing DNS scheme is just adding overhead without added privacy. If a DNS scheme hides the client's identity from the recursive resolver, it satisfies the property Hides-Client-Identity-from-Resolver.

P5 Hides-Client-Identity-in-Stage-2: In Stage-2, there are two types of entities that may invade clients' privacy: ASes between the recursive resolver and the ANSes, and ANSes that the recursive resolver queries when it is traversing the DNS hierarchy. Although it is not necessary, in Stage-2, recursive resolvers typically send the complete QName to the ANSes, which reveals the client's requested QName to all of the traversed ANSes. Besides, if the client includes ECS in DNS queries, the recursive resolvers in Stage2 also include ECS in their iterative queries. Recursive resolvers that support ECS will propagate ECS in their queries to ANSes as they are traversing the DNS hierarchy. Hence, the middle entities in Stage-2 and the ANSes (from root to leaf) will have access to the client's included ECS. If a scheme deletes or truncates ECS from the client's query to preserve clients' privacy in Stage-2, it Hides-Client-Identity-in-Stage2. Hence, this property is provided by minimizing client-related information in Stage- 2 . In the fourth column of Table 4.1, ( $\checkmark$ ) demonstrates a condition in which QName minimization (see 2.1.4) is not applied and ECS (see 2.1.3) is included in the DNS queries. Therefore, due to the inclusion of ECS in $(\checkmark)$, Hides-Client-Identity-in-Stage-2 is satisfied if a scheme by design deletes ECS from the queries. 
P6 Resilient-to-Eavesdropping-in-Stage-1: Due to the lack of confidentiality in DNS, DNS queries and responses are susceptible to eavesdropping. Despite being a security property, we categorize confidentiality of DNS queries and responses as a privacy property. Our rationale follows, DNS information is not secret; it is inherently public. Despite that, transmitting DNS in plain-text would harms clients' privacy. Confidentiality is achieved by encrypting the transmitted DNS messages, and confidentiality must be added to both of name resolution stages to restrict access of unauthorized parties to message content. Lack of confidentiality decreases clients' privacy in both stages [92].

In Stage-1, if the traffic between a client and recursive resolver is encrypted, the DNS alternative is Resilient-to-Eavesdropping-in-Stage-1. Therefore, middle ASes, such as ISPs, will not have access to the content of the DNS messages. However, the recursive resolver still has access to the DNS query and client's IP address and P4 is required to hide client's identity from the resolver.

P7 Resilient-to-Eavesdropping-in-Stage-2: In Stage-2, if ECS is included in DNS messages and QName minimization is not applied, due to the disclosed client-related information, confidentiality becomes more significant. Similar to Stage-1, if traffic between recursive resolvers and ANSes is encrypted, the scheme is Resilient-to-Eavesdropping-

in-Stage-2, and ASes between recursive resolvers and ANSes will not have access to DNS message content. However, the endpoints in Stage-2 (i.e., ANSes) still have access to the DNS messages.

\subsection{Threat Models}

\subsubsection{Assumptions}

When rating a DNS scheme, we assume that it is implemented correctly. A broken implementation can affect a scheme's security in practice. For instance, Chung et al. [25] showed 
that a large portion of DNS entities are not implementing DNSSEC properly, with vulnerabilities leading to broken chains of trust. In practice, a large number of DNSSEC entities do not participate properly, which affects the security and availability of the other parties [25].

Another assumption is that the client uses a remote recursive resolver (i.e., cloud-based or public resolver, instead of the ISP's resolver) because the paths to these resolvers contain the intermediate entities in the local network and ISP. Additionally, the path to these remote resolvers also goes through different ASes between the stub- and recursive- resolver over the Internet. The case of a remote resolver is considered as the worst case, as they have a larger attack surface than the two other scenarios (local or ISP).

\subsubsection{Compromising Privacy Versus Anonymity}

We use the terms privacy and anonymity as follows.

Privacy. Privacy relates to the protection of a user's identity on personally identifiable information (PII) in the DNS messages [77]. As such, we consider properties such as Hides-Client-Identity-from-Resolver, and Hides-Client-Identity-in-Stage-2, which reflect a scheme's ability to hide a client's IP address from the resolver/ANS as privacy properties. Additionally, eliminating ECS from queries is considered a privacy technique.

Anonymity. In the DNS context, unlike privacy, anonymity is hiding user's behaviors/actions. Therefore, properties such as Mystifies-Message-Nature-in-Stage-1, ConcealsMessage-Nature-in-Stage-1, or Resists-ISP-Correlation-Analysis are considered as anonymity properties. QName minimization (Sec. 2.1.4) is considered an anonymity mechanism, as it reduces disclosure of a client's actions in Stage-2.

\subsubsection{Threats}

We consider two main motivations for DNS attacks: Corrupting DNS responses for traffic redirection (i.e., active attacks), and eavesdropping on DNS for compromising privacy 
and/or anonymity (i.e., passive attacks). Corruption is used to mount MitM or censorship; eavesdropping for surveillance, user or device identification, or user profiling. Other attacks such as DRDoS exploit the DNS infrastructure to amplify the effectiveness of DDoS attacks, and target the availability of entities over the Internet. Regarding privacy problems, as explained in RFC-7626 [19], due to the lack of encryption in DNS, intermediate eavesdroppers can have access to the queried QNames and client's IP address in both name resolution stages. Additionally, recursive resolvers and ANSes, as a part of the name resolution process, are able to gather and process client-related data [19]. Due to the lack of authentication, fraudulent resolvers and ANSes can also cause privacy and security threats by gathering client-related information or redirecting clients to malicious destinations [19]. Cache snooping attacks reveal privacy-relevant data about the clients that are using a specific recursive resolver [83, 19].

Security threats, such as DNS hijacking or DNS cache poisoning, are actively affecting the name resolution process. In the absence of data origin authentication, DNS hijacking occurs-when the authorized parties in a domain name resolution procedure cannot validate the integrity and authenticity of the messages they receive, entities in the middle of a name resolution can alter the DNS records or messages for malicious purposes, leading to pharming attacks [76]. Tampering can be made locally on the client-side by malware, in intermediate entities, or by ANSes, which affects a large number of clients.

On the other hand, DNS cache poisoning occurs when an attacker injects false DNS records into the DNS caches. As a result of cache poisoning at Stage-2, the recursive resolver's cache will be filled with incorrect DNS records. Non-cryptographic techniques, such as source port and transaction ID randomization, proposed to add 32-bit random nonce to the DNS queries to make the resolvers resilient against blind cache poisoning attacks. However, these methods are not effective against novel side-channel based cache poisoning attacks [66]; an intermediate entity that has access to the transmitted DNS messages and can eavesdrop on the nonces [50]. Data origin authentication $(\mathrm{S} 8, \mathrm{~S} 9)$ is a sufficient condition 


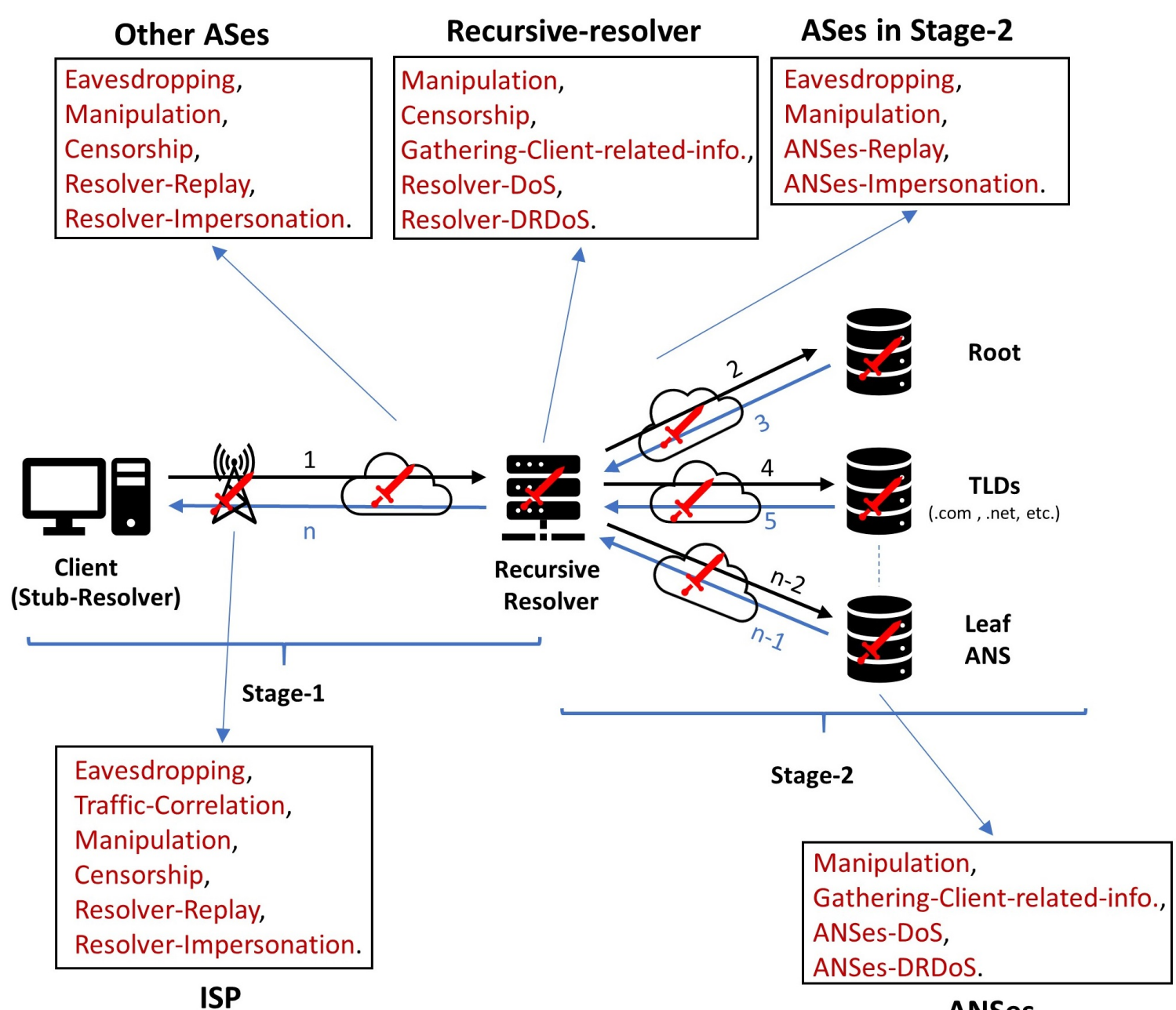

ISP

ANSes

Figure 4.1 DNS entities that can be targets for attacks (blue), and the type of attacks on these (red).

to mitigate network-based cache poisoning attacks.

Figure 4.1 provides a summary of the attacks that can occur due to lack of security, and privacy. Attacks in the figure are categorized into 5 groups based on the entities that can pose threats to a client's name resolution procedure. These entities are assumed free of malware but capable of performing the listed attacks. In Stage-1, the ISP, other ASes, and the recursive resolver can pose active and passive attacks to threaten the client's privacy or security. In Stage-2, different ASes and ANSes are involved in processing and/or delivering DNS traffic, and thus exposes the name resolution process to potential risks. Governments 
can coerce ISPs and ASes to perform passive and active attacks on DNS messages [11]. ANSes can also act maliciously based on their operator's decisions. Therefore, ISPs, ASes, and ANSes are not considered as trusted and harmless entities.

In addition to the attacks that target the client, DoS and DRDoS attacks target recursive resolver's and ANSes' availability. The security and availability threats are considered active attacks, and the privacy and anonymity threats are considered passive attacks. In Figure 4.1 Eavesdropping, Gathering-Client-Related-info., and Traffic-Correlation are passive, and the other threats are considered as active threats.

\subsection{Schemes Evaluation}

We can create an evaluation framework based on the security, availability, privacy, and anonymity properties defined in sections 4.1 and 4.2. We thus place these properties in a table, Table 4.1, where columns are the properties and rows are secure DNS schemes. Each cell in Table 4.1 will have a bullet $(\bigcirc)$, a half-circle $(\bigcirc)$, or an empty circle ( $\bigcirc)$. A bullet in row $i$ column $j$ means the scheme in row $i$ satisfies the property in column $j$. Half-circle means the scheme in row $i$ partially satisfies column $j$ property, and an empty circle means the scheme fails to satisfy the property.

\subsection{Rating Vanilla DNS}

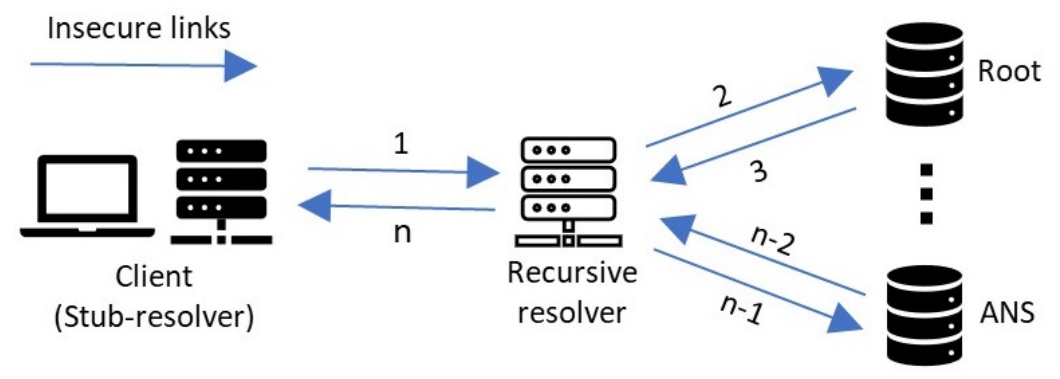

Figure 4.2 Vanilla-DNS: provides none of the properties in the framework. 
None of the defined security properties in Section 4.1 had been considered as part of the original DNS protocol. Vanilla DNS is vulnerable to all of the attacks, and privacy and anonymity threats, that may occur as a consequence to the lack of security. The only satisfied security property for Vanilla DNS is the Hides-Client-Identity-in-Stage-2 if the client does not include ECS as part of the query, and the resolver applies QName minimization (see Table 4.1). In that case, no client-identity data will be disclosed in Stage-2. Vanilla DNS uses UDP protocol (except for the messages that exceed the defined maximum size), and is thus vulnerable to flooding and DRDoS amplification attacks.

\subsection{A Survey of Secure-DNS Alternatives}

We survey secure-DNS schemes, classifying each in three categories based on their commonly proposed working stage in practice. Some of these schemes have been designed to secure Stage-1, others Stage-2. A small subset targets both name resolution stages. We briefly explain these protocols, and rate them using the framework explained in the following section (Sec. 4.4).

\subsubsection{DNSSEC}

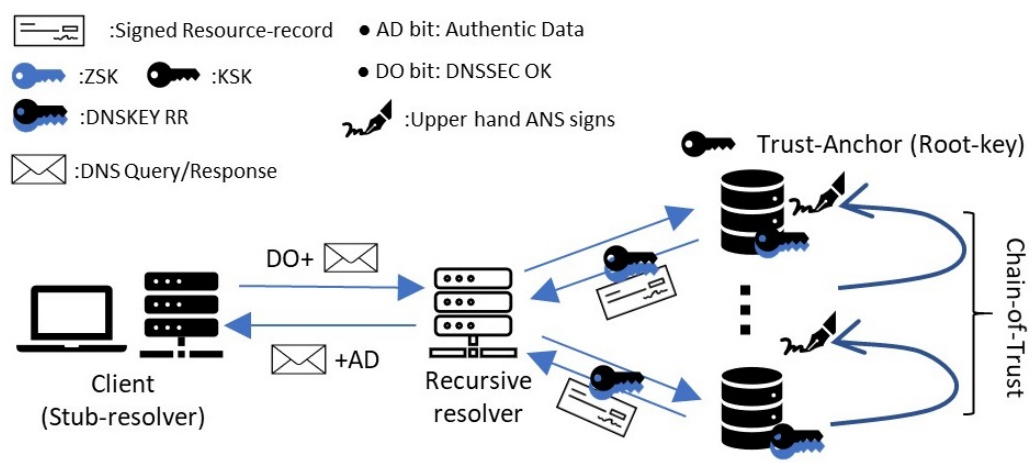

Figure 4.3 DNSSEC: Designed to provide end-to-end integrity and data origin authentication.

As the latest IETF documents of DNSSEC (i.e., RFCs 4033-4035) describe, DNSSEC 
(Figure 4.3) adds a set of security extensions to provide authenticated denial of existence, integrity, and data origin authentication to the DNS responses from the ANSs $[8,10,9]$.

DNSSEC adds two pairs of public keys to the ANSes: Key-Signing-Key (KSK) and Zone-Signing-Key (ZSK) [8]. The private part of ZSK is used to sign the Resource Records (RRs) in a zone. RRs are different record types in a DNS zone (e.g., 'A' record for IPv4 or 'PTR' records for reverse DNS lookups). There is a specific DNSKEY RR in DNSSEC that contains the public part of both KSK and ZSK; this specific record is signed by the private part of KSK. Hash of DNSKEY record known as Delegation Signer (DS) will be sent to the parent zone to sign and publish. The chain of these DS records sent to the parent zones will create a chain of trust to the root zone, which is considered as the trust anchor in DNSSEC. Thus, ANSes rely on their parent authoritative name servers to establish the chain of trust until the root name servers $[27,4]$.

As Figure 4.3 illustrates, the security-aware client (i.e., stub-resolver) sends a recursive query (Recursion Desired bit set 'RD=1') to the recursive name server with the DNSSEC Okay 'DO' bit set. Security-aware stub-resolver is a stub-resolver that understands DNSSEC related bits, but does not validate signatures on its own. Instead, it relies on the recursive resolver to verify DNSSEC keys and signatures. If the recursive resolver supports DNSSEC, when the resolver queries the ANSes, it receives the DS and DNSKEY records RR from the ANSes. Then the recursive resolver validates the signatures and keys that it receives from the ANSes. If the validation succeeds, the resolver sets the Authenticated Data 'AD' bit and sends the response to the stub-resolver (client).

In this procedure, although the stub-resolver is "security-aware" (meaning it checks for the AD bit), it trusts the recursive resolver and the network on which it transfers the DNS messages (i.e., Stage-1) [8]. In DNSSEC, security-aware stub-resolvers can also be validating. These validate the signatures and keys voluntarily, instead of trusting the recursive resolver and the middle network entities in Stage-1. Unfortunately, the majority of stubresolvers in practice are non-validating $[53,8]$. Although the portion of validating stub- 
resolvers increased in 2019 [53], it remains that less than a third of clients world-wide are validating stub-resolvers. As such, DNSSEC is mainly used in Stage-2. Another problem of DNSSEC is the added overhead in the DNS traffic and the significantly increased amplification factor, which could be used in DRDoS amplification attacks [27].

DNSSEC does not provide properties related to Stage-1, which makes it vulnerable to attacks that target the communication network and entities in Stage-1. Regarding availability, it is not Resilient-to-Resolver-DoS, Resilient-to-ANS-DoS, and Resilient-to-DRDoS, as in both stages it relies on UDP, and the amplification ratio is high. DNSSEC only provides Resilient-to-Manipulation-in-Stage-2 and Resilient-to-ANS-Impersonation in Stage-2 using RR signatures. As anyone can replay DNSSEC messages when the signatures are valid, it is not Resilient-to-ANS-Replay-Attack. Finally, DNSSEC fails to provide any of the privacy and anonymity properties as it was not designed for that. It only provides Hides-ClientIdentity-in-Stage-2 when the ECS extension is not used.

\subsubsection{DNSCurve}

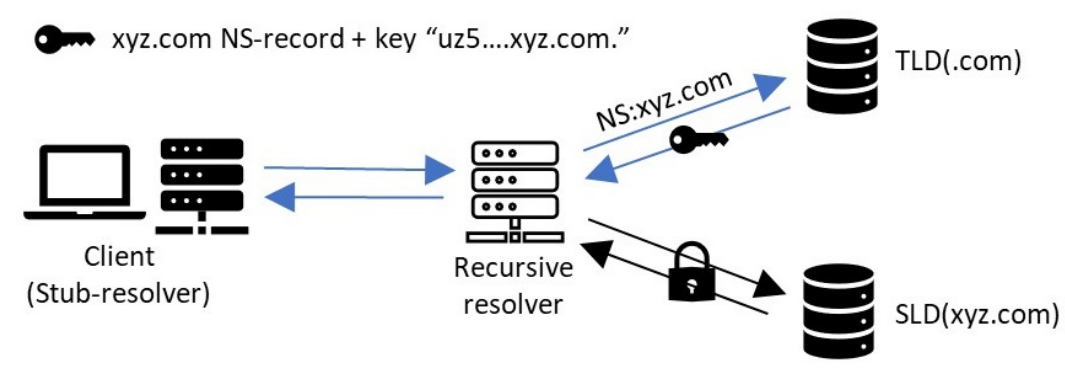

Figure 4.4 DNSCurve: Only adds security properties to the communication between the resolver and ANSes.

DNSCurve uses public-key encryption with Elliptic Curve Cryptography (ECC) to add security properties to Stage-2, and typically uses port 53 on the ANSes side [15]. DNSCurve is faster than DNSSEC because of the shorter keys in ECC and smaller DNS message lengths [4]. As Figure 4.4 illustrates, when the recursive resolver queries the NS record 
of the zone from the parent zone, the NS answers with a concatenated 54-byte label containing its public key (e.g., carleton.ca. NS uz5nh8m56...lq7rpj81.carleton.ca.) [29]. The public key is 51-bytes, encoded in base-32 and comes after (hard coded) string ' $u z 5$ '. This string indicates that an ANS supports DNSCurve. When the resolver sends a query to a DNSCurve-supporting ANS, it includes its own public key in that query, and encrypts the query with the ANS's public key, and signes it with its (resolver's) private key.

Key exchange is achieved by Curve25519, and the Salsa20 stream cipher will use the generated session-key to provide confidentiality and mitigating eavesdropping in Stage$2[15,14]$. Poly1305 MAC is used to provide message authentication and resilience to manipulation $[75,15]$. However, it is unclear which scenario the secure session between recursive resolver and ANSes is required. ${ }^{1}$ For the requests and responses, resolvers and ANSes generate 96-bit nonces. Two ways are recommended in the documentation to generate nonces: using timestamps as the first 64-bits or using counters (sequence-number) as the first 64-bits of nonces [29]. Therefore, nonces in the cryptosystem of DNSCurve can provide freshness, and we assume liveness (in specific time windows) to mitigate replay attacks.

None of the Stage-1 security properties is satisfied in DNSCurve. The amplification ratio of (responses/queries) in DNSCurve is approximately 1. Therefore, it is not beneficial to the adversaries to perform amplification DRDoS attacks against ANSes in DNSCurve. Additionally, unlike DNSSEC, DNSCurve does not require significant changes to DNS messages and the zones file [4]. However, it requires changes to the ANSes and resolvers. DNSCurve does not inform the client regarding the validity of the responses-clients have to blindly trust the recursive resolver to implement and use DNSCurve properly [4].

DNSCurve is Resilient-to-Eavesdropping-in-Stage-2, using encryption, also Resilientto-Manipulation-in-Stage-2 and Resilient-to-ANS-Impersonation, using MAC. Although it

\footnotetext{
${ }^{1}$ To the best of our knowledge, authoritative information about DNSCurve exists only at dnscurve.org and [29] (expired dreaft). No known research paper or RFC standard exist for DNSCurve.
} 
is not explicitly mentioned in the documentation [29], DNSCurve uses nonces that can be used with time windows to achieve Resilient-to-ANS-Replay-Attack. DNSCurve typically works on top of UDP, and thus is not Resilient-to-ANS-DoS and Resilient-to-Resolver$D o S$ [15]. However, the amplification ratio for the ANSes in DNSCurve is low $(\sim 1)$, and it is partially Resilient-to-DRDoS (i.e., only for ANSes and not recursive resolver). Similar to DNSSEC, DNSCurve satisfies P5 when ECS is not included in the queries, and does not actively delete or truncate ECS. Finally, DNSCurve is designed to work in Stage-2, thus provides none of the Stage-1 properties.

\subsubsection{DNSCrypt*V3:}

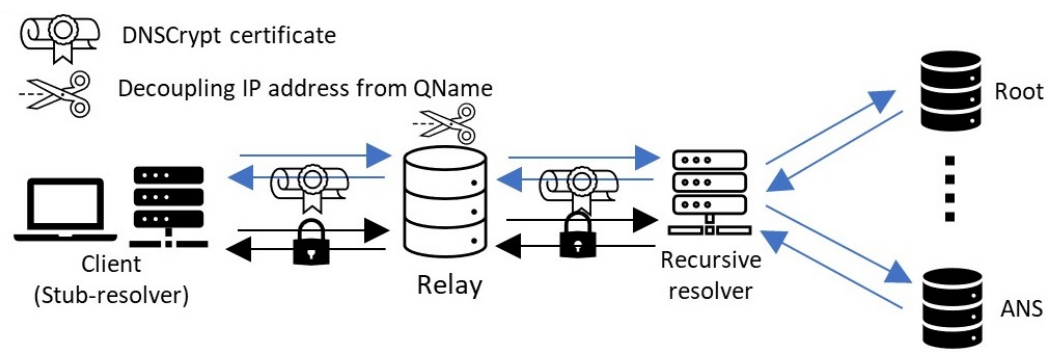

Figure 4.5 DNSCrypt*V3: Provides security properties in Stage-1, plus using relay server to enhance clients' privacy and anonymity.

Similar to DNSCurve, DNSCrypt uses public-key encryption with ECC. However, DNSCrypt secures Stage-1 of the domain name resolution. As Figure 4.5 shows, firstly, the client queries a DNSCrypt resolver for its certificate. DNSCrypt does not rely on the Internet's PKI, and the client must know the resolver's name and public key to verify the certificates and authenticate the resolver [31]. DNSCrypt certificates are encoded as a TXT record, and have a specific structure mentioned in its documentation [31]. The client uses the certificate parameters to establish a secure communication with the DNSCrypt resolver.

Key exchange in DNSCrypt is performed using Curve25519 elliptic curve and hSalsa20 hash function [31]. The shared secret is then used by XSalsa20 stream cipher with Poly1305 message authentication mechanisms to transfer messages with confidentiality, integrity, 
and authenticity [31]. Additionally, DNSCrypt includes 24-bytes nonce in queries and responses, and it is recommended in its documentation to include timestamps as part of the nonces for freshness and liveness. To increase privacy, DNSCrypt uses ISO/IEC 7816-4 format to add specific and variable bytes for padding the transmitted messages [31]. It runs over port 443, merging with web traffic, thus making it less distinguishable. Detecting DNSCrypt messages is costly for intermediate entities, as they have to fingerprint and analyze traffic and destination addresses. In DNSCrypt version 3, sending anonymized queries is added to this protocol. As Figure 4.5 shows, unlike DNS-over-Tor, which adds layers of encryption and overhead, DNSCrypt uses an intermediate relay server to forward the encrypted DNS messages [30]. As a result, the relay server does not have access to the queried domain name, and the resolver does not have access to the client's IP address, ensuring the client's privacy and anonymity with a low overhead [30]. In practice, a list of relay servers is maintained by community volunteers. ${ }^{2}$ Similar ODoH single-layer proxies [72], if the owner of the relay is the same as the owner of the resolver (or if they collude), this one-layer anonymization technique fails [91]. If ECS is included in the client's DNS queries, the anonymizing technique also fails. Finally, DNSCurve can work over both TCP and UDP [31].

DNSCrypt is Resilient-to-Eavesdropping-in-Stage-1 using encryption; Resilient-to-Manipulationin-Stage-1, and Resilient-to-Resolver-Impersonation using MAC. Resilient-to-Resolver-ReplayAttack is achieved by nonces, which contain timestamps. Merging with web traffic in DNSCrypt Mystifies-Message-Nature-in-Stage-1, and it partially provides Resists-ISP-CorrelationAnalysis. Censoring its port number thus blocks the entire HTTPS traffic, and censoring by the port is not practical; hence, DNSCrypt is Resilient-to-Port-based-Censorship. Moreover, finding resolvers' IP addresses using only port scanners is not feasible, and DNSCrypt is Resilient-to-IP-based-Censorship. Additionally, as DNSCrypt provides S1, S8, and P6 properties, it is Resilient-to-Exclusive-QName-Censorship. DNSCrypt traffic Using DNSCrypt over TCP (especially with the SYN cookies and rate-limiting) provides

\footnotetext{
${ }^{2}$ https://github.com/DNSCrypt/dnscrypt-resolvers/blob/master/v3/relays.md
} 
Resilient-to-Resolver-DoS. Besides, the amplification ratio in DNSCrypt is low, thus it is not beneficial to the adversaries to use it for DRDoS and partially provides Resilient-to$D R D o S$ for the resolvers. Additionally, the property Hides-Client-Identity-from-Resolver is achieved by the intermediate relays.

\subsubsection{DNS-over-TLS (DoT)}

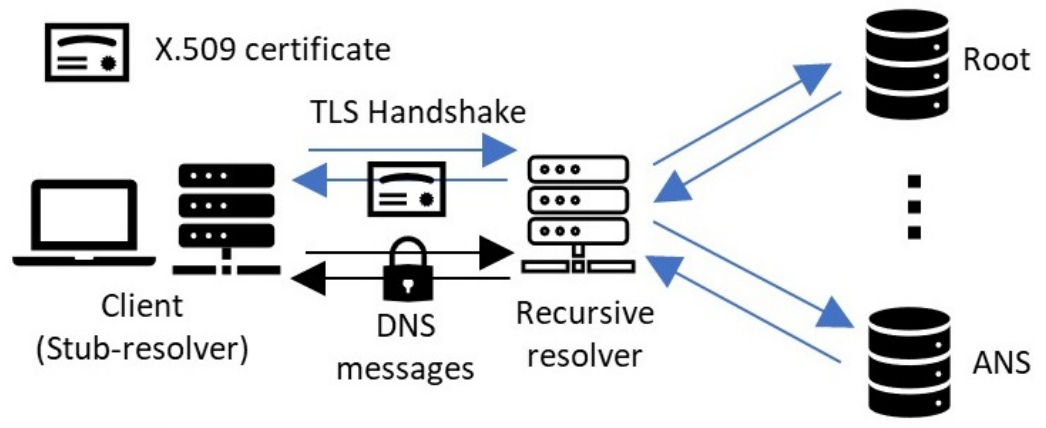

Figure 4.6 DoT: Sending DNS queries over TLS in Stage-1.

DoT uses TLS to transfer the DNS queries in Stage-1 [49, 109]. Figure 4.6 illustrates DoT's working procedure. A client initiates a TLS handshake with a DoT resolver, and verifies the resolver's certificate using PKI trust anchors. Trust anchors might vary based on stub-resolvers and their operating system. Similar to HTTPS, DoT uses X.509 certificates to bind the public key of resolvers to their domain names. Relying on the PKI means DoT is susceptible to fraudulent certificates issued by compromised CAs. After the TLS handshake, client and resolver use a secure session with a shared-key to transfer DNS messages [49]. DoT provides two modes: strict and opportunistic. In the opportunistic mode, DoT makes security checks only if possible [49]. Otherwise, it will fallback to Vanilla DNS. In the strict mode, successful authentication and encryption are mandatory to resolve a domain name.

Based on the used TLS cipher-suites, DoT provides resolver authentication (S6), message confidentiality (P6), integrity (S1), and data origin authentication (S8). As investi- 
gated in Ch. 3, approximately a third of the DoT certificates were invalid (e.g., self-signed, expired) [63].

When DoT is used with persistent TLS connection, it adds negligible overhead compared to Vanilla DNS [63]. But the excessive number of open connections can exhaust the resolver resources. Regarding anonymity, DoT does not provide any means to hide the client's IP address from the resolvers, and the resolvers have access to the clients' QNames. DoT suggests using the EDNS(0) padding option to pad the DNS messages with a variable number of octets and make them more resilient against traffic-analysis and side-channel leaks in Stage-1. However, recent literature shows that encryption and padding techniques in DoT are not strong enough to resist Machine Learning (ML) based traffic analysis attacks [93, 48]. DoT uses a specific port (853) and its traffic is distinguishable, which makes its traffic susceptible to blocking, redirecting, or censorship [49, 63].

DoT is Resilient-to-Manipulation-in-Stage-1, Resilient-to-Eavesdropping-in-Stage-1, Resilientto-Resolver-Impersonation, and Resilient-to-Resolver-Replay-Attack all provided by TLS. Additionally, it provides Resilient-to-Exclusive-QName-Censorship by integrity, data origin authentication and confidentiality. However, because of its dedicated port, DoT is neither Resilient-to-Port-based-Censorship nor Resilient-to-IP-based-Censorship. Regarding resolvers' availability, DoT uses TCP, and is thus Resilient-to-Resolver-DoS and partial Resilient-to-DRDoS (only for the resolvers).

\subsubsection{DNS-over-HTTPS (DoH)}

DoH relies on HTTPS (i.e., get and post) with Uniform Resource Identifiers (URIs) to transfer DNS messages (e.g., https://dnsserver.example.net/dns-query?dns) [46]. It provides similar security properties to DoT in Stage-1. The resolvers' domain name is bound to their public keys via X.509 certificates that are signed by CAs. As such, similar to DoT, DoH inherits PKI limitations. However, unlike DoT, DoH does not have the opportunistic mode, 


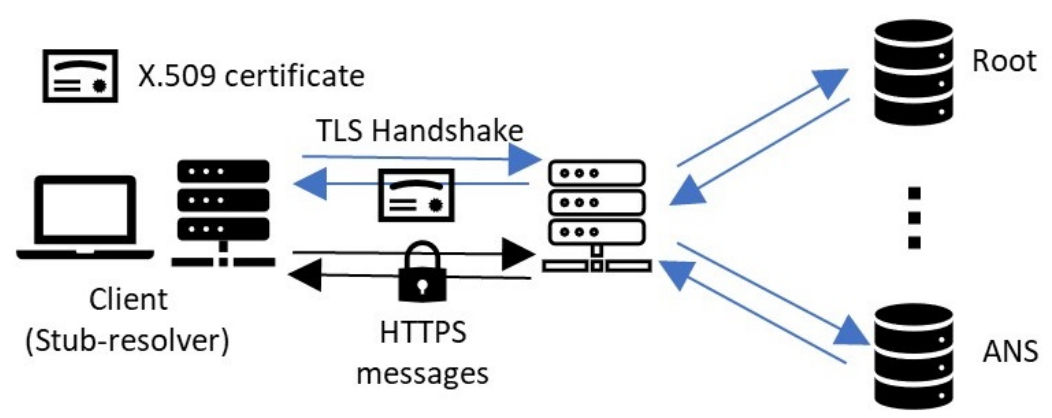

Figure 4.7 DoH: Sending DNS queries over HTTPS using URIs and HTTP methods

so it does not fail open in case of invalid certificates. If certificate validation fails, name resolution fails. As a result of the strict security in DoH, Lu et al. [63] (in 2019) did not find DoH resolvers with invalid certificates. Also, DoH uses the same port (443) as HTTPS, merging DNS traffic with web traffic, and its traffic is thus less distinguishable [63, 46].

DoH suggests HTTP/2 compression, besides the EDNS(0) DNS padding, to enhance the privacy of the transmitted messages and make the DoH traffic robust against traffic analysis and side-channel leaks [46, 68]. However, similar to DoT, Siby et al. [93] proved that EDNS(0) padding does not make a scheme resilient to ML-based traffic analysis attacks, but it makes traffic analysis more costly for the adversaries. Lastly, as DoH works over TCP, it can use SYN cookies with rate-limiting techniques to improve the availability of resolver [109].

DoH is Resilient-to-Manipulation-in-Stage-1, Resilient-to-Eavesdropping-in-Stage-1, Resilientto-Resolver-Replay-Attack, and Resilient-to-Resolver-Impersonation. DoH Mystifies-MessageNature-in-Stage-1 and Resilient-to-Port-based-Censorship by merging its traffic with web over port 443, and consequently satisfies Resists-ISP-Correlation-Analysis. It is not possible to discover DoH resolvers by scanning its port over the Internet and censor their IP addresses; hence, DoH is also Resilient-to-IP-based-Censorship. As DoH works on top of TCP, it is Resilient-to-Resolver-DoS and partial Resilient-to-DRDoSfor the resolvers. DoH is Resilient-to-Exclusive-QName-Censorship by providing confidentiality, integrity 
and message authentication. Overall, regarding security, DoH is significantly similar the DoT. However, due to the mystification of DNS traffic in DoH, clients' privacy is enhanced, and DoH is more resilient against censorship.

\subsubsection{DNS-over-QUIC (DoQ)}

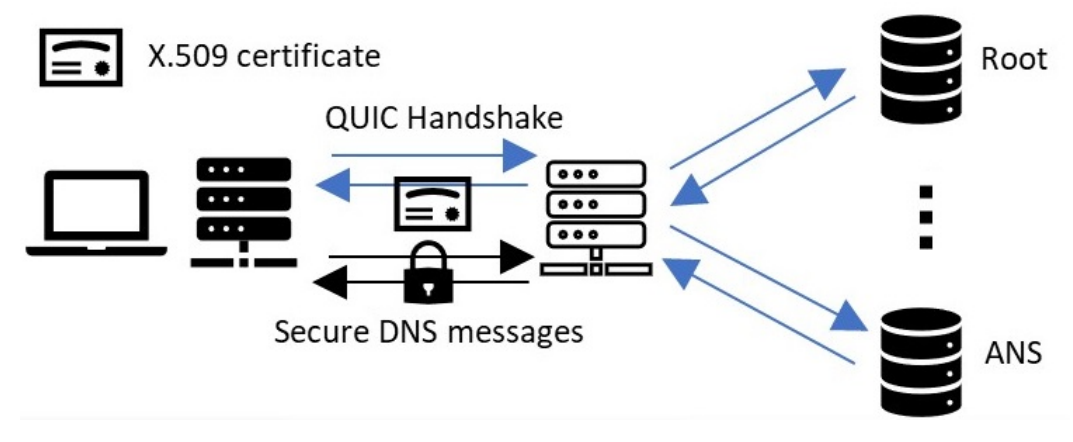

Figure 4.8 DoQ: Sending DNS using QUIC transport protocol.

DNS-over-Quick (DoQ) uses QUIC transport protocol to decrease the handshake latency of TLS in Stage-1 [51]. DoQ provides similar security properties to DoT, with some additional enhancements through multiplexing mechanisms. As Figure 4.8 illustrates, DoQ workflow is similar to DoT, and the only difference is the faster handshake between client and resolver. QUIC provides low-latency connection establishment and better error correction mechanisms through Forward Error Corrections (FEC), which allows QUIC to recover lost packets from other packets and the FEC packet $[54,51]$. DoQ adds confidentiality, integrity, and authentication to Stage-1. DoQ uses QUIC padding to increase the encrypted DNS messages' privacy [51]. However, as it has been shown for DoT and DoH, encryption and straightforward paddings do not make DNS resilient to traffic analysis attacks [93], and DoQ thus remains weak against ML-based traffic analysis. DoQ uses a distinguishable port number, experimentally port (784), and its traffic is easily distinguishable in Stage- 1.

Similar to DoT, DoQ is Resilient-to-Manipulation-in-Stage-1, Resilient-to-Eavesdroppingin-Stage-1, Resilient-to-Resolver-Replay-Attack, Resilient-to-Resolver-Impersonation, and Resilient-to-Exclusive-QName-Censorship. However, due to using a specific port, DoQ is 
not Resilient-to-Port-based-Censorship, Resilient-to-IP-based-Censorship, and MystifiesMessage-Nature-in-Stage-1. Another disadvantage of DoQ is using UDP as the transport protocol. Although UDP decreases latency, DoQ resolver is vulnerable to the flooding and reflection DoS attacks, as the resolver does not have sufficient information to detect spoofed packets [64]. Therefore DoQ recursive resolvers in Stage-1 have not been provided with means to achieve Resilient-to-Resolver-DoS and Resilient-to-DRDoS properties.

\subsubsection{DNS-over-DTLS (DoDTLS)}

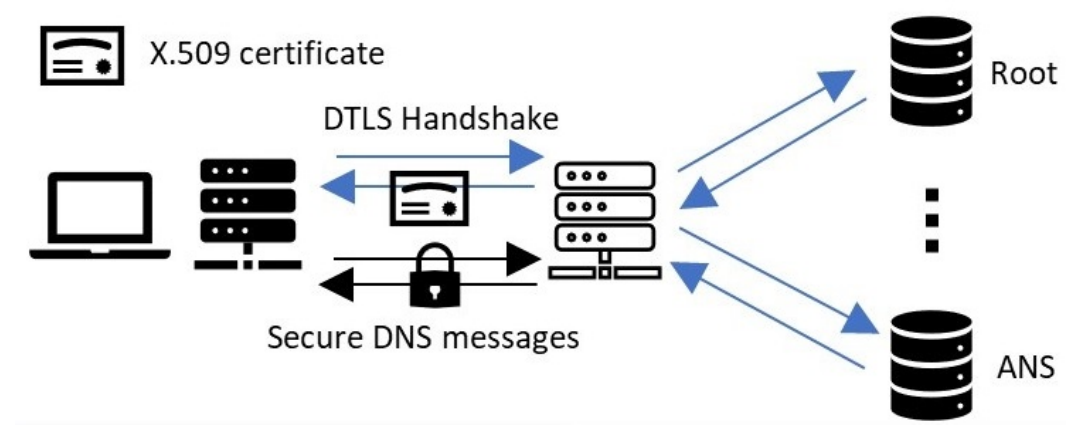

Figure 4.9 DoDTLS: Sending DNS using DTLS transport protocol.

As proposed in RFC-8094, DNS-over-DTLS (DoDTLS) uses Datagram Transport Layer Security (DTLS) to secure Stage-1 DNS messages. Unlike DoT that works over TCP, DoDTLS uses UDP to decrease latency. The only difference between DoT and DoDTLS is the handshake, which is a stateless version of TLS hanshake with additional packet fields for error handeling and retransmission of the messages [86]. DoDTLS uses the same port as DoT (853), and provides the same security properties, namely server authentication, message confidentiality, integrity, and authentication. As DoDTLS relies on Internet's PKI for authentication, it also adds similar TLS PKI problems and complexities to the DNS ecosystem. Similar to DoT, EDNS(0) padding could be implemented in the DoDTLS. However, encryption and simple padding schemes will not significantly improve the privacy of the DNS messages, and DoDTLS is also considered susceptible to the traffic analysis attacks [93]. 
DoDTLS does not implement mechanisms to anonymize a client's IP address from the recursive resolver. Hence, DoDTLS resovlers have access to the clients' IP addresses and QNames. Similar to other UDP-based alternatives, the insufficient means in UDP to avoid DoS, DoDTLS cannot assure the availability of the recursive-resolvers in Stage- 1 .

As another variant of TLS, DoDTLS provides Resilient-to-Manipulation-in-Stage-1, Resilient-to-Eavesdropping-in-Stage-1, and Resilient-to-Exclusive-QName-Censorship. It is also Resilient-to-Resolver-Replay-Attack and Resilient-to-Resolver-Impersonation. DoDTLS uses a specific port; its traffic is distinguishable and it does not Mystifies-Message-Naturein-Stage-1. As DoDTLS is a Stage-1 scheme, it does not provide any security properties in Stage- 2 .

\subsubsection{DNS-over-Tor (DoTor)}

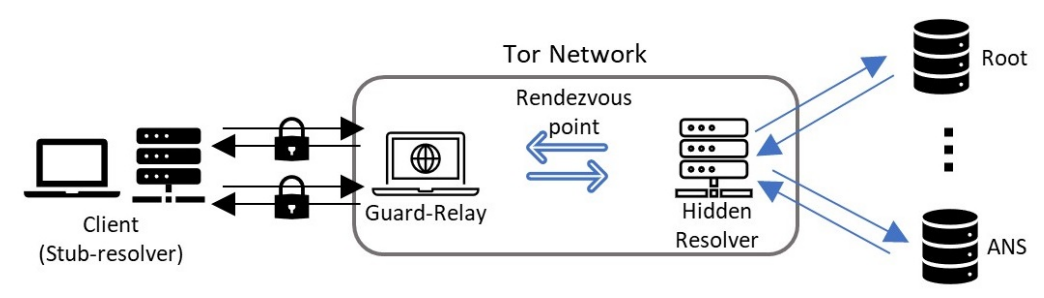

Figure 4.10 DNS-over-Tor: Based on Cloudflare's hidden resolver structure [89].

Figure 4.10 illustrates DoTor working procedure. Firstly, the client establishes a circuit with the hidden resolver through the onion routers including the rendezvous point [34]. Then, the DNS traffic gets encrypted by the exchanged keys with the middle relays and resolver's key, and is sent through Tor network to the hidden resolver. The entry node to the Tor network that the client directly communicates with is called Guard-relay. The hidden resolver will traverse the hierarchy of DNS insecurely, and send the response through Tor's onion tunnel to the client. Tor is vulnerable to different types of attacks $[41,104,81]$. One example is a traffic correlation, where entry and exit traffic can be analyzed to reveal the client's identity. If the client uses the domain name of the recursive resolver instead of its 
IP address, the likelihood of a successful correlation attack increases [41]. Bad relays [81] are another problem, which can perform different types of manipulation and redirection to damage anonymity or manipulate a client's traffic $[81,104]$. To decrease the correlation attack surface, DoTor resolver can be implemented as a '.onion' service, which also mitigates the DefecTor attacks (where the adversaries analyze the exit relay name resolution for correlation attacks) [89, 41]. By using a '.onion' recursive resolver, name resolution will not take place at the exit nodes, rather inside Tor network, thus mitigating Defector attack [41]. In April 2018, Cloudflare implemented hidden DNS-over-Tor (DoTor) resolver. ${ }^{3}$

DoTor hides DNS messages with layers of encryption that enhances the privacy of the client against the recursive-resolver and Stage-1 entities (i.e., ISP, ASes) [34]. Thus, ISPs cannot distinguish DNS traffic, nor correlate it to reveal requested domain names. Additionally, Siby et al. [93] reported that the only secure-DNS scheme that is significantly resilient against ML-based traffic analysis attacks, is DoH-over-Tor. However, layers of encryption and TLS communications between relays can significantly increase the time and size overhead compared to other alternatives, and Tor naturally sacrifices latency for security. Depending on the user/application, the security benefits of sending DNS messages over Tor can outweigh the overhead that DoTor causes [72]. As Tor uses TCP, Tor nodes can implement SYN cookies and rate-limiting.

DoTor is the only protocol that Conceals-Message-Nature-in-Stage-1 with layers of encryption, thus it is also Mystifies-Message-Nature-in-Stage-1, Resilient-to-Eavesdroppingin-Stage-1, and Resists-ISP-Correlation-Analysis. Tor intermediate nodes provide HidesClient-Identity-from-Resolver. Resolvers in DoTor, are Resilient-to-Resolver-DoS and Resilientto-DRDoS using TCP. Additionally, transmitted messages between the client and hidden resolver provide integrity using hash functions, and DoTor provides Resilient-to-Manipulationin-Stage-1. The hidden resolver authenticates itself to the client by its public key, which is also used to generate the 'onion' domain name. Additionally, using short-term ephemeral

\footnotetext{
${ }^{3}$ Cloudflare's DoTor resolver supports DoT, DoH, and Vanilla-DNS over Tor.
} 
keys in Tor circuits mitigates replay attacks-So DoTor is Resilient-to-Resolver-ReplayAttack. However, Tor entry relays can get blocked by IP; hence, DoTor is not Resilient-to-IPbased-Censorship. Tor relays can use arbitrary ports (that can be used by other protocols, e.g., 443), thus blocking Tor relays using port number is infeasible, and DoTor is Resilientto-Port-based-Censorship. Finally, DoTor is a Stage-1 scheme and does not provide any property in Stage-2.

\subsubsection{Oblivious DNS (ODNS)}

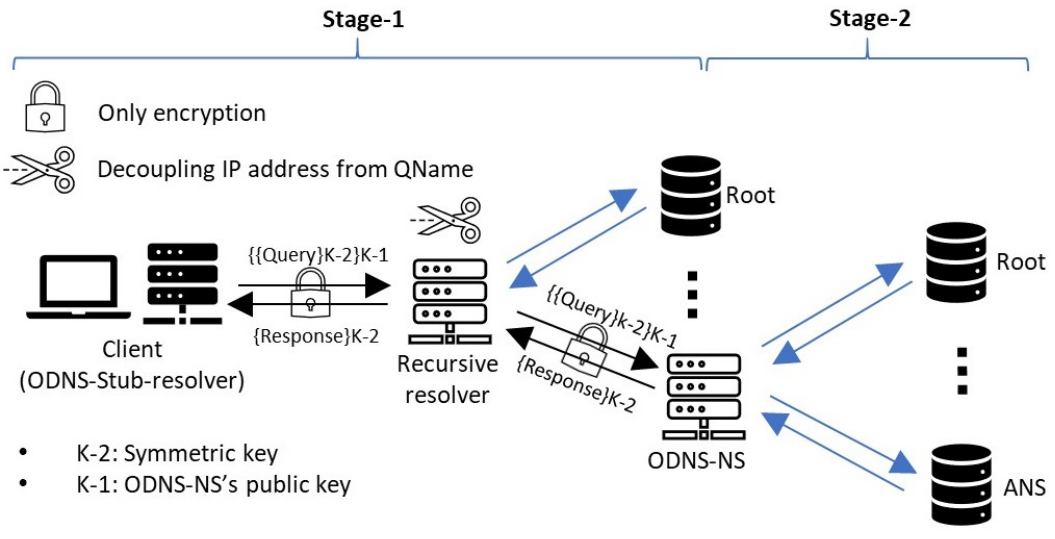

Figure 4.11 Oblivious DNS: Adding confidentiality and Privacy to the DNS queries [91].

Proposed by Schmitt et al. [91], ODNS is designed to protect clients' privacy and anonymity from the recursive resolvers by decoupling a client's IP address from the queried domain name. An ODNS resolver will not have access to the client's IP address, and the recursive resolver in Stage-1 will not have access to the client's queried domain name. It works on Stage-1, and causes only a negligible overhead [91]. Before name resolution, to get ODNSNS's public key $K-1$, a client has to send a special query to the recursive resolver, and the recursive resolver anycasts this query to ODNS-NSs and receives the nearest ODNSNS's public key included in the EDNS extensions. Finally, the recursive resolver sends the ODNS-NS's key to the client. As Figure 4.11 illustrates, a client encrypts DNS queries with a symmetric key $K-2$, and then encrypts $K-2$ with $K-1$. The query is sent to the 
recursive resolver, and the recursive resolver forwards the DNS query with its IP address to the ODNS-NS, which is a recursive name server. At this point, the ODNS-NS will traverse the hierarchy of the DNS to resolve the queried domain name, encrypts the final response with the client's symmetric key $K-2$, and send it back to the client.

In Stage-1, the middle entities between the client and recursive resolver know the IP but not the query, and the entities between the recursive resolver and ODNS-NS neither know the query nor the client's IP address. ODNS removes ECS from the DNS queries in the ODNS stub-resolvers to preserve client's privacy [91]. So, even if the client adds its IP address to the queries in the ECS, an ideal ODNS stub-resolver forwards the query without it. Therefore, ODNS hides the client's identity from the ODNS-NS and Stage-2 entities.

ODNS encrypts the QNames in Stage-1 and thus provides Resilient-to-Eavesdroppingin-Stage-1. This scheme also Hides-Client-Identity-from-Resolver, using a one-layer proxy. ODNS is the only protocol that stresses on the importance of eliminating ECS at the client; it provides Hides-Client-Identity-in-Stage-2. ODNS does not provide any property in Stage-2 as it is proposed for Stage-1. Also, due to the lack of integrity and message authenticity, ODNS is not Resilient-to-Exclusive-QName-Censorship, Resilient-to-Manipulation-inStage-1, and Resilient-to-Resolver-Impersonation. Besides, due to the distinguishable port number, it is not Resists-ISP-Correlation-Analysis. Similar to Vanilla DNS-ODNS is not Resilient-to-IP-based-Censorship and Resilient-to-Port-based-Censorship [72].

\subsubsection{Confidential DNS}

In order to provide confidentiality through encryption in both of the name resolution stages, Confidential DNS adds a new RR 'ENCRYPT', to the recursive resolvers and ANSes [103].The 'ENCRYPT' RR contains a public key for the resolver or ANS, which is used to exchange a shared-secret or a public key that will be uesd for DNS query and response [103]. Confidential DNS works in two modes: opportunistic and authenticated. In the former, the client does 


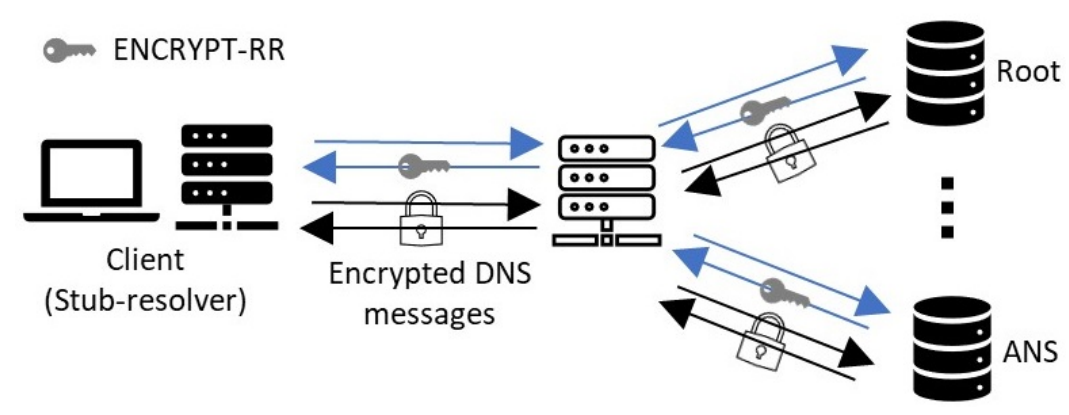

Figure 4.12 Confidential-DNS: Adding confidentiality in both stages.

not authenticate the identity of the server. In the latter, the client authenticates the server by validating the RR signatures 'RRSIGs' provided by DNSSEC [103]. As a result, Confidential DNS typically provides opportunistic encryption (i.e., Provides encryption when it is possible, otherwise, fallback to plain-text) without authentication, and adds authentication on top of DNSSEC [103]. The authors [103] also mentioned that the authenticity of the DNS messages, which have been provided by DNSSEC, are not among the primary goals of confidential DNS.

In Table 4.1, we evaluate opportunistic Confidential DNS without DNSSEC. Confidential DNS is only Resilient-to-Eavesdropping-in-Stage-1 and Resilient-to-Eavesdropping-inStage-2. Regarding privacy, Confidential DNS does not use anonymizing techniques, so does not satisfy Hides-Client-Identity-from-Resolver. It is not clear If Confidential DNS uses a port different from 53. If Confidential DNS uses the same port as Vanilla DNS, its traffic becomes distinguishable from others, thus can be blocked or censored. In rating we assume it uses the same port as Vanilla DNS. Additionally, due to the lack of authenticity and integrity, this scheme is not Resilient-to-Exclusive-QName-Censorship. Confidential DNS uses UDP, and is thus not Resilient-to-Resolver-DoS, Resilient-to-ANS-DoS, and Resilientto-DRDoS. 
Table 4.1 Comparative evaluation framework of secure-DNS alternatives

\begin{tabular}{|c|c|c|c|c|c|c|c|c|c|c|c|c|c|c|c|c|c|c|c|c|c|c|}
\hline DNS Scheme & 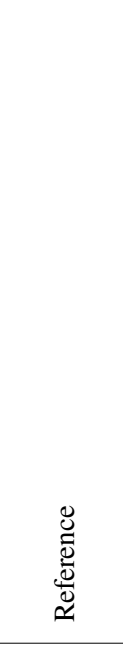 & 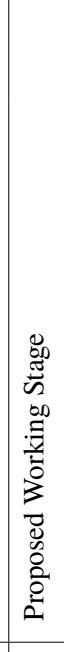 & 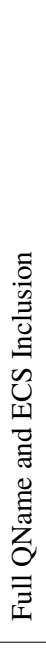 & 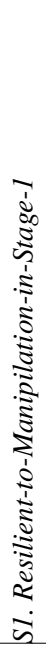 & 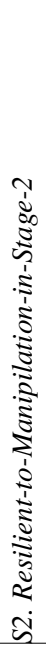 & 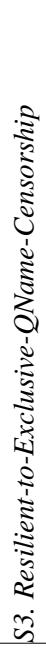 & 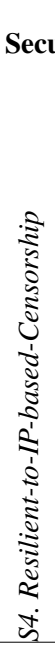 & 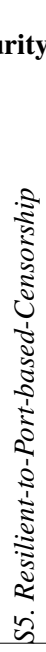 & 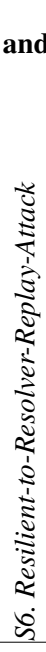 & 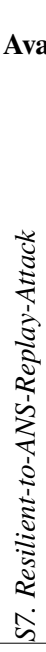 & 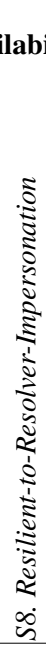 & 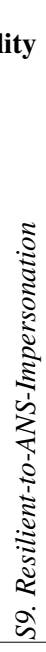 & 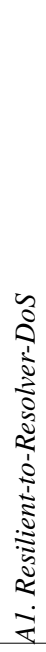 & 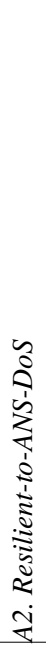 & 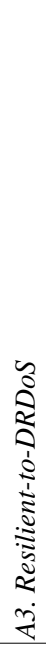 & 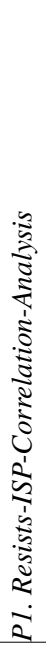 & 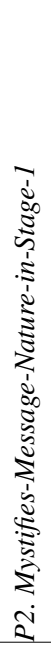 & 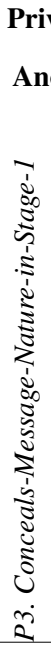 & 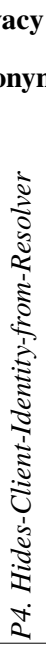 & 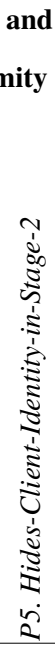 & 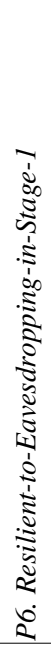 & 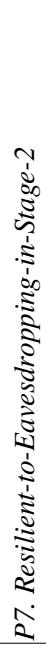 \\
\hline Vanilla DNS & {$[70,69]$} & $1 \& 2$ & $\begin{array}{l}\checkmark \\
x\end{array}$ & $\begin{array}{l}0 \\
0\end{array}$ & $\begin{array}{l}0 \\
0\end{array}$ & $\begin{array}{l}0 \\
0\end{array}$ & $\begin{array}{l}\bigcirc \\
\bigcirc\end{array}$ & $\begin{array}{l}0 \\
0\end{array}$ & $\begin{array}{l}0 \\
0\end{array}$ & $\begin{array}{l}0 \\
0\end{array}$ & $\begin{array}{l}0 \\
0\end{array}$ & $\begin{array}{l}0 \\
0\end{array}$ & $\begin{array}{l}0 \\
0\end{array}$ & $\begin{array}{l}\bigcirc \\
0\end{array}$ & $\begin{array}{l}0 \\
0\end{array}$ & $\begin{array}{l}\bigcirc \\
0\end{array}$ & $\begin{array}{l}0 \\
0\end{array}$ & $\begin{array}{l}0 \\
0\end{array}$ & $\begin{array}{l}\bigcirc \\
0\end{array}$ & $\begin{array}{l}0 \\
0\end{array}$ & $\begin{array}{l}0 \\
0\end{array}$ & $\begin{array}{l}0 \\
0\end{array}$ \\
\hline DNSSEC & {$[8,10,9]$} & & $\begin{array}{l}\checkmark \\
x\end{array}$ & $\begin{array}{l}0 \\
0\end{array}$ & 0 & $\begin{array}{l}0 \\
0\end{array}$ & $\begin{array}{l}0 \\
0\end{array}$ & $\begin{array}{l}0 \\
0\end{array}$ & $\begin{array}{l}0 \\
0\end{array}$ & $\begin{array}{l}0 \\
0\end{array}$ & $\begin{array}{l}0 \\
0\end{array}$ & 0 & $\begin{array}{l}0 \\
0\end{array}$ & $\begin{array}{l}0 \\
0\end{array}$ & $\begin{array}{l}0 \\
0\end{array}$ & $\begin{array}{l}0 \\
0\end{array}$ & $\begin{array}{l}0 \\
0\end{array}$ & $\begin{array}{l}0 \\
0\end{array}$ & $\begin{array}{l}\bigcirc \\
0\end{array}$ & & $\begin{array}{l}0 \\
0\end{array}$ & $\begin{array}{l}0 \\
0\end{array}$ \\
\hline DNSCurve & [15] & & $\begin{array}{l}\checkmark \\
x\end{array}$ & $\begin{array}{l}0 \\
0\end{array}$ & & $\begin{array}{l}0 \\
0\end{array}$ & $\begin{array}{l}\bigcirc \\
\bigcirc\end{array}$ & $\begin{array}{l}0 \\
0\end{array}$ & $\begin{array}{l}0 \\
0\end{array}$ & 0 & $\begin{array}{l}0 \\
0\end{array}$ & 0 & $\begin{array}{l}0 \\
0\end{array}$ & $\begin{array}{l}0 \\
0\end{array}$ & $\begin{array}{l}\mathbf{1} \\
\mathbf{0}\end{array}$ & $\begin{array}{l}0 \\
0\end{array}$ & $\begin{array}{l}0 \\
0\end{array}$ & $\begin{array}{l}0 \\
0\end{array}$ & $\begin{array}{l}0 \\
0\end{array}$ & & $\begin{array}{l}0 \\
0\end{array}$ & \\
\hline DNSCrypt*V3 & {$[31,30]$} & & $\begin{array}{l}\checkmark \\
x\end{array}$ & 0 & $\begin{array}{l}0 \\
0\end{array}$ & & 0 & & & $\begin{array}{l}0 \\
0\end{array}$ & 0 & $\begin{array}{l}0 \\
0\end{array}$ & & $\begin{array}{l}0 \\
0\end{array}$ & $\begin{array}{l}\mathbf{1} \\
\mathbf{1}\end{array}$ & $\mathbf{0}$ & & $\begin{array}{l}0 \\
0\end{array}$ & & & & $\begin{array}{l}0 \\
0\end{array}$ \\
\hline DNS-over-TLS & [49] & & $\begin{array}{l}\checkmark \\
x\end{array}$ & & $\begin{array}{l}0 \\
0\end{array}$ & O & $\begin{array}{l}\bigcirc \\
0\end{array}$ & $\begin{array}{l}0 \\
0\end{array}$ & 0 & $\begin{array}{l}0 \\
0\end{array}$ & 0 & $\begin{array}{l}0 \\
0\end{array}$ & 0 & $\begin{array}{l}0 \\
0\end{array}$ & $\begin{array}{l}0 \\
0\end{array}$ & $\begin{array}{l}\bigcirc \\
0\end{array}$ & $\begin{array}{l}0 \\
0\end{array}$ & $\begin{array}{l}0 \\
0\end{array}$ & $\begin{array}{l}0 \\
0\end{array}$ & & & $\begin{array}{l}0 \\
0\end{array}$ \\
\hline DNS-over-HTTPS & [46] & & $\begin{array}{l}\checkmark \\
x\end{array}$ & & $\begin{array}{l}\bigcirc \\
0\end{array}$ & 0 & 0 & 0 & 0 & $\begin{array}{l}0 \\
0\end{array}$ & 0 & $\begin{array}{l}0 \\
0\end{array}$ & 0 & $\begin{array}{l}0 \\
0\end{array}$ & $\begin{array}{l}\mathbf{1} \\
\mathbf{1}\end{array}$ & $\mathbf{0}$ & & $\begin{array}{l}0 \\
0\end{array}$ & $\begin{array}{l}0 \\
0\end{array}$ & & & $\begin{array}{l}0 \\
0\end{array}$ \\
\hline DNS-over-DTLS & [85] & 1 & $\begin{array}{l}\checkmark \\
x\end{array}$ & & $\begin{array}{l}0 \\
0\end{array}$ & 0 & $\begin{array}{l}\bigcirc \\
\bigcirc\end{array}$ & $\begin{array}{l}0 \\
0\end{array}$ & & $\begin{array}{l}0 \\
0\end{array}$ & 0 & $\begin{array}{l}0 \\
0\end{array}$ & $\begin{array}{l}0 \\
0\end{array}$ & $\begin{array}{l}0 \\
0\end{array}$ & $\begin{array}{l}0 \\
0\end{array}$ & $\begin{array}{l}0 \\
0\end{array}$ & $\begin{array}{l}0 \\
0\end{array}$ & $\begin{array}{l}0 \\
0\end{array}$ & $\begin{array}{l}0 \\
0\end{array}$ & & & $\begin{array}{l}0 \\
0\end{array}$ \\
\hline DNS-over-QUIC & {$[51]$} & & $\begin{array}{l}\checkmark \\
x\end{array}$ & 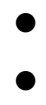 & $\begin{array}{l}\bigcirc \\
\bigcirc\end{array}$ & 0 & $\begin{array}{l}\bigcirc \\
\bigcirc\end{array}$ & $\begin{array}{l}\bigcirc \\
\bigcirc\end{array}$ & 0 & $\begin{array}{l}\bigcirc \\
\bigcirc\end{array}$ & ○ & $\begin{array}{l}\bigcirc \\
\bigcirc\end{array}$ & $\begin{array}{l}\bigcirc \\
\bigcirc\end{array}$ & $\begin{array}{l}\bigcirc \\
\bigcirc\end{array}$ & $\begin{array}{l}0 \\
0\end{array}$ & $\begin{array}{l}\bigcirc \\
\bigcirc\end{array}$ & $\begin{array}{l}0 \\
0\end{array}$ & $\begin{array}{l}0 \\
0\end{array}$ & $\begin{array}{l}\bigcirc \\
\bigcirc\end{array}$ & $\bigcirc$ & & $\begin{array}{l}\bigcirc \\
\bigcirc\end{array}$ \\
\hline DNS-over-Tor & [89] & & $\begin{array}{l}\checkmark \\
x\end{array}$ & O & $\begin{array}{l}0 \\
0\end{array}$ & $\begin{array}{l}\bigcirc \\
\bigcirc\end{array}$ & $\begin{array}{l}\bigcirc \\
\bigcirc\end{array}$ & 0 & ? & $\begin{array}{l}0 \\
0\end{array}$ & $\begin{array}{l}0 \\
0\end{array}$ & $\begin{array}{l}0 \\
0\end{array}$ & 0 & $\begin{array}{l}0 \\
0\end{array}$ & $\begin{array}{l}\mathbf{1} \\
\mathbf{1}\end{array}$ & . & & & & & & $\begin{array}{l}0 \\
0\end{array}$ \\
\hline Oblivious DNS & [91] & & $\begin{array}{l}\checkmark \\
x\end{array}$ & $\begin{array}{l}0 \\
0\end{array}$ & $\begin{array}{l}\bigcirc \\
\bigcirc\end{array}$ & $\begin{array}{l}\bigcirc \\
\bigcirc\end{array}$ & $\begin{array}{l}\bigcirc \\
\bigcirc\end{array}$ & $\begin{array}{l}\bigcirc \\
\bigcirc\end{array}$ & $\begin{array}{l}0 \\
0\end{array}$ & $\begin{array}{l}\bigcirc \\
\bigcirc\end{array}$ & $\begin{array}{l}\bigcirc \\
\bigcirc\end{array}$ & $\begin{array}{l}\bigcirc \\
\bigcirc\end{array}$ & $\begin{array}{l}0 \\
0\end{array}$ & $\begin{array}{l}\bigcirc \\
\bigcirc\end{array}$ & $\begin{array}{l}0 \\
0\end{array}$ & $\begin{array}{l}\bigcirc \\
\bigcirc\end{array}$ & $\begin{array}{l}0 \\
0\end{array}$ & $\begin{array}{l}0 \\
0\end{array}$ & 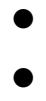 & $\bullet$ & 0 & $\begin{array}{l}\bigcirc \\
\bigcirc\end{array}$ \\
\hline Confidential DNS & {$[103]$} & $1 \& 2$ & $\begin{array}{l}\checkmark \\
x\end{array}$ & $\begin{array}{l}0 \\
0\end{array}$ & $\begin{array}{l}\bigcirc \\
\bigcirc\end{array}$ & $\begin{array}{l}\bigcirc \\
0\end{array}$ & $\begin{array}{l}\bigcirc \\
\bigcirc\end{array}$ & $\begin{array}{l}\bigcirc \\
0\end{array}$ & $\begin{array}{l}0 \\
0\end{array}$ & $\begin{array}{l}0 \\
0\end{array}$ & $\begin{array}{l}0 \\
0\end{array}$ & $\begin{array}{l}0 \\
0\end{array}$ & $\begin{array}{l}0 \\
0\end{array}$ & $\begin{array}{l}\bigcirc \\
0\end{array}$ & 0 & $\begin{array}{l}0 \\
\bigcirc\end{array}$ & $\begin{array}{l}0 \\
0\end{array}$ & $\begin{array}{l}0 \\
0\end{array}$ & $\begin{array}{l}\bigcirc \\
\bigcirc\end{array}$ & $\bigcirc$ & 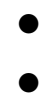 & D \\
\hline
\end{tabular}




\subsection{Insights from the Table}

We provide insights from our DNS evaluation regarding association between properties, and comparisons across schemes.

\subsubsection{Association Among Properties}

Before discussing the bigger picture in Table 4.1, we clarify the association and difference between properties that have similar ratings. Although similarities can imply redundancy between properties, they can also be an artifact of the number of rated schemes. When possible, where a similarity exists, we will explain a hypothetical DNS scheme that would achieve one property but not the other.

\section{Resilient-to-Manipulation-in-Stage-1, Resilient-to-Resolver-Impersonation, and Resilient-} to-Exclusive-QName-Censorship. The rating in all three columns is similar. Despite that, the properties are not the same. Both Resilient-to-Manipulation-in-Stage-1 and Resilientto-Resolver-Impersonation are necessary for a scheme to be Resilient-to-Exclusive-QNameCensorship, but are insufficient on their own. We believe any scheme that is also Resilientto-Eavesdropping-in-Stage-1 may automatically become Resilient-to-Exclusive-QName-Censorship. Regarding the similarity between Resilient-to-Manipulation-in-Stage-1 and Resilient-to-ResolverImpersonation, the former is achieved by integrity mechanisms, while the latter is a result of data origin authentication. Data origin authentication is considered as a stronger subset of integrity, so every data origin authentication also includes integrity [67]. For instance, if a scheme just uses hash digests for data integrity, it satisfies Resilient-to-Manipulation-inStage-1 but not Resilient-to-Resolver-Impersonation.

\section{Resilient-to-Port-based-Censorship and Mystifies-Message-Nature-in-Stage-1. Based} on the definitions, there is a correlation between these two properties. When a scheme Mystifies-Message-Nature-in-Stage-1, its traffic gets merged with other traffic, and portbased censoring will also censor another protocol. For instance, in DoH, port-based cen- 
sorship also shuts down web traffic. Although merging traffic is a sufficient condition for Mystifies-Message-Nature-in-Stage-1, but it is not necessary. A scheme that employs traffic-flow security techniques can also mystify the DNS traffic nature, and such scheme is Mystifies-Message-Nature-in-Stage-1 but not Resilient-to-Port-based-Censorship.

Resilient-to-Resolver-Replay-Attack and Resilient-to-Resolver-Impersonation. The former property is achieved by entity authentication, and the latter by data origin authentication. Data origin authentication is a necessary condition for entity authentication. In addition to data origin authentication, entity authentication also requires a means for liveness and freshness. In our evaluation, if a scheme provides freshness, we assume that it can also provide liveness based on that freshness. For instance, if a scheme provides data origin authentication (e.g., with MAC) but does not provide means for freshness and liveness (e.g., timestamps), this scheme will be Resilient-to-Resolver-Impersonation but not Resilient-to-Resolver-Replay-Attack. We found no such scheme in our set, which explains the similarities between both properties in the table.

Resilient-to-Manipulation-in-Stage-2 and Resilient-to-ANS-Impersonation. Resilientto-Manipulation-in-Stage-2 is achieved if a scheme provides means to ensure integrity, and Resilient-to-ANS-Impersonation is achieved by data origin authentication. For our purposes herein, data origin authentication provides means to both: detect alterations by attackers and verify the source that originated the message; we thus consider data origin authentication to be a (stronger) subset of integrity. Therefore, a scheme can provide integrity (e.g., using hash functions) without data origin authentication, and satisfy Resilient-to-Manipulationin-Stage-2 without Resilient-to-ANS-Impersonation. Again, we found no such scheme in our set.

Resilient-to-Resolver-DoS and Resilient-to-DRDoS. The former protects the resolver itself from flooding attacks; the latter protects entities other than the resolver over the Internet, when the adversaries leverage the resolver to reflect and amplify DoS attacks. Both properties can be satisfied if TCP is used. Usage of TCP is a sufficient but not necessary 
condition for a scheme to be Resilient-to-DRDoS. The primary motive for a DRDoS attack is the amplification of DDoS traffic. Thus, DRDoS could be made less rewarding to attackers by decreasing the amplification factor. If a DNS scheme has a $1: 1$ ratio for queries and responses over UDP, DRDoS attacks would not be beneficial to adversaries, and such scheme would thus be Resilient-to-DRDoS but not Resilient-to-Resolver-DoS.

\subsubsection{Comparison of Schemes}

A few interesting notes can be made from Table 4.1. First, none of the secure-DNS schemes provide security in the whole path of the name resolution (both stages), and none satisfy all properties. While five schemes are Resilient-to-Manipulation-in-Stage-1, only DNSSEC and DNSCurve provide Resilient-to-Manipulation-in-Stage-2.

TLS-based schemes. Among the TLS-based schemes (i.e., DoT, DoH, DoDTLS, DoQ), DoH is the most secure as it is resilient to all censorship-related properties, provides MystifiesMessage-Nature-in-Stage-1, and partial Resists-ISP-Correlation-Analysis. DoDTLS and DoQ satisfy fewer security properties than the others.

Near-identical schemes. DNSCrypt*V3 and DoH provide the same properties, except for Hides-Client-Identity-from-Resolver; DoH does not provide any means for this property, but DNSCrypt uses an intermediate relay to decouple the client's IP address from its query.

Basic schemes. Confidential DNS only adds a single property (i.e., Resilient-to-Eavesdropping) in both stages. ODNS adds three properties: two in Stage-1 (i.e., Resilient-to-Eavesdroppingin-Stage-1 and Hides-Client-Identity-from-Resolver), and Hides-Client-Identity-in-Stage-2.

Confidentiality of DNS messages. All of the secure-DNS schemes that work in Stage1 provide confidentiality in that stage; hence, they are all Resilient-to-Eavesdropping-inStage-1. However, in Stage-2, only DNSCurve and Confidential-DNS provide confidentiality. That is, in the majority of secure-DNS alternatives, DNS traffic in Stage-2 is typically accessible by unauthorized parties. If it contains sensitive information such as ECS or com- 
plete QName, it will disclose privacy or anonymity-related data about clients in Stage-2. That highlights the importance of eliminating or truncating ECS from DNS messages, and employing QName minimization to minimize the impact on clients' privacy and anonymity.

Censorship resilience. Schemes that use a distinguishable port number are susceptible to port-based censorship. Only three are using ports that merge their traffic with other protocols: DNScrypt, DoH, DoTor. It can also be noticed that schemes that are susceptible to port-based censorship are also susceptible to IP-based censorship, as all of their resolvers could be discovered using an efficient Internet scanner like Zmap [37]. Furthermore, as the Tor guard relays are limited, and their IP addresses are listed publicly, governments can censor DNS-over-Tor by blocking the IP addresses of the Tor guard relays. Overall, DNSCrypt and DoH appear the most resilient to censorship.

Availability. Regarding availability of recursive-resolver, only Stage-1 schemes that work on top of TCP are Resilient-to-Resolver-DoS and Resilient-to-DRDoS. However, none of the rated schemes protects ANSes against DoS attacks. One possible reason is that the number of Stage-2 schemes is limited, and ANSes can be unwilling to accept stateful protocols that increase their overhead.

Privacy champions. Three of the secure-DNS alternatives satisfy Mystifies-MessageNature-in-Stage-1, namely DNSCrypt, DoH, and DoTor. However, only DoTor Conceals DNS message nature. Therefore, only DoTor protects against ISP-correlation attacks; the two other schemes (i.e., DoH and DNSCrypt) only make such attacks costly. Overall, the majority of DNS alternatives do not provide means of mystifying or concealing the nature of the DNS messages. The only scheme that traded latency for privacy was ODNS, which proposed using ECS with zero value in the ODNS stub-resolvers. All of the schemes with Resilient-to-Resolver-Replay-Attack also have Resilient-to-Resolver-Impersonation. Table 4.1 shows that in Stage-1, only TLS-based schemes plus DNSCrypt provide these two properties. Regarding Stage-2 of the name resolution, only DNSCurve provides means to achieve Resilient-to-ANS-Replay-Attack. 
Summary. As the results show, Stage-1 protocols mainly provide integrity, confidentiality, and message authentication in that stage. However, these protocols typically have privacy deficiencies against intermediate entities and recursive resolver. Regarding Stage-2 protocols, we can observe that if ECS is truncated or removed from the queries, and QName is minimized, no client-related data is accessible in Stage-2. However, data origin authentication is a necessary property in Stage-2 to mitigate ANS impersonation and cache poisoning attacks.

\subsection{Discussion}

One general observation is that the schemes designed to work in Stage- 2 are limited, and provide only a handful of the defined security benefits. On the other hand, Stage-1 schemes are diverse, and provide various combinations of properties. We now discuss several points upon surveying and rating secure-DNS schemes.

Persistence of Vanilla DNS in practice. Vanilla DNS remains the dominant scheme for name resolution in practice. DNSSEC and DNSCurve were proposed to remedy Vanilla DNS's vulnerabilities. However, they also lack security properties, and suffer from limited deployability after years of being proposed.

The privacy benefits of TLS-based schemes are questionable. TLS-based schemes (i.e., DoT, DoH, DoQ, DoDTLS) mainly provide security properties, but they typically do not provide privacy and anonymity. As the Internet's PKI evolved, the infrastructure that TLS-based schemes rely on provides sufficient security in their ecosystem. As any recursive resolver and client can implement these alternatives without requiring other entities to make any changes, these schemes have less deployability barriers. Nevertheless, such schemes have been proposed only to provide security in Stage-1. ANSes are expected to be reluctant to implement these schemes in Stage-2 due to increased CPU usage and a significant increase in transmitted DNS traffic. To have a secure name resolution procedure, a 
Stage-2 scheme is required to protect the DNS messages in Stage-2.

Stronger privacy in other alternatives. Transmitting DNS messages over the Tor network (DoTor) provides privacy and anonymity benefits more than the other alternatives in Stage-1 (i.e., P1, P2, P3, P4, and P6). The only scheme that offers strong privacy against ISP is DoTor, which conceals the nature of traffic, and the ISP cannot detect that a name resolution is occurring (in Table 4.1: DNS-over-Tor is the only scheme with full bullets for P1 and P3). To provide security benefits alongside privacy and anonymity, DoTor can be combined with other compatible schemes (e.g., DoH-over-Tor). ODNS and ODoH use a layer of proxy to provide similar properties to DoTor with lower overhead and latency. However, they are susceptible to censorship and correlation attacks. Finally, Confidential DNS only provides opportunistic encryption to DNS messages. The security properties that Confidential DNS provides are limited, and its authenticated mode relies on DNSSEC, which is not to date widely adopted.

ISP correlation. ISPs can perform reverse DNS lookups to find out the previously encrypted resolved domain name when the client sends out the web traffic over TLS. Although reverse IP lookup might not reveal the exact domain name, clients using the Server Name Indication (SNI) TLS extension that will leak the exact domain name to the ISP [40]. For example, if the ISP observes a traffic over port 853, the ISP would understand that an encrypted DoT name resolution is occurring, and if the subsequent traffic is an HTTPS traffic, the ISP can reverse lookup its destination IP address or check the SNI field in the TLS client-hello message. Additionally, DNS traffic could be used by the ISP or recursive resolver alongside the IP-ID field to identify the traffic coming from behind a Network Address Translation (NAT) [78].

Complete availability. Availability is a necessary security property for both resolvers and ANSes to guarantee that a name resolution takes place faultlessly. In Stage-1, the recursive resolver must be available to the client, and in Stage-2, the ANSes must be available to the recursive resolver. Hence, if any of the mentioned entities do not have the property of 
availability, legitimate clients cannot resolve domain names successfully (if the DNS record is not cached), and the schemes are not resilient against DoS.

Deployability issues. Due to the DNS's two-staged structure and participation of different entities in the name resolution procedure, proposing a comprehensive DNS scheme that provides security properties in both stages can be feasible. However, complex DNS alternatives that require significant changes are unlikely to gain wide-scale adoption, or the possibility of ANSes being potentially reluctant to change (e.g., in adoption of DNSSEC [25] or DNSCurve), or add CPU/bandwidth overhead, large-scale adoption of Stage-2 schemes is still an open challenge.

Combining schemes. Combining schemes is a potential solution to address security deficits in DNS. For instance, Cloudflare combines DoH, or DoT with the Tor network to provide strong privacy, anonymity, availability, and security in Stage-1 [89]. Another example is Oblivious DoH $(\mathrm{ODoH})$, which combines ODNS with DoH to enhance clients' privacy, implemented by Cloudflare and Apple [60,94]. Additionally, ODNS is compatible with DoT and DNSSEC [91]. Confidential DNS works with DNSSEC to authenticate the ENCRYPT RR and work in the authenticated mode [103]. TLS-based schemes (i.e., DoT, DoH, DoDTLS, and DoQ) appear to, not only be compatible with DNSSEC, but also complement it.

Centralization. It is widely accepted that relying on a single recursive resolver is harmful to clients' privacy $[47,91]$. As mentioned in Section 4.1, the client's accumulated name resolution history can be used for many different purposes. Therefore, secure-DNS schemes such as DoTor or DNSCrypt have been proposed with mechanisms to hide the identity (i.e., IP address) of clients from the resolvers. Further, new mechanisms such as ODNS and DoH with ODNS (ODoH) have also been proposed to decouple the client's IP address from the queried domain name, thus hiding the identity of the client from the recursive resolvers with negligible overhead $[60,94,91]$. These anonymizing mechanisms can be used compatibly with the other secure-DNS schemes. Hoang et al. [45] proposed K-resolver to 
distribute DNS queries among different DoH resolvers to avoid the accumulation of client queries in one resolver, which mitigates the harms caused by resolver-centralization. 


\section{Chapter 5}

\section{Conclusion and Future Work}

This thesis shows that a long way remains ahead in secure and private DNS research. Challenges can be exacerbated by different stakeholders in the DNS ecosystem with different interests, which might be conflicting. Addressing their interests can pose its own set of challenges. Regarding security, availability, privacy, and anonymity aspects, we observe that all of the surveyed secure-DNS schemes provide only a subset of properties.

Designing a scheme that satisfies all the security and privacy properties of our framework (Ch. 4) appears achievable. However, deployability and usability barriers are expected to rise against such a scheme. A scheme is bound to fail if it does not address deployability challenges, or does not incentivize different entities in the DNS ecosystem to adopt it.

We show that combining different schemes is a promising at hand solution to the security and privacy deficiencies of DNS. However, the DNS story is not near its end, and future schemes have to consider security, usability, and deployability in their design to observe significant enhancements in the DNS ecosystem worldwide. 


\subsection{Future Work}

As our future work, we investigate deployability properties such as Backward-Compatibility, Incremental-Deployability, requirements of Zone-File-Change, Resolver-Change, ClientSide-Change, and Requring-New-Infrastructure. We also plan to incorporate usability properties such as Low-Latency and Ease-of-Use in our framework. The multi-facet assessment of secure-DNS alternatives would be helpful for different users to choose the best alternatives that suit their needs. Additionally, we will evaluate the DoT client-side tools from usability and security facets, such as Uses-Key-Pinning, Certificate-Security-Checks, Configurability, and Ease-of-Use. 


\section{Bibliography}

[1] Josh Aas, Richard Barnes, Benton Case, Zakir Durumeric, Peter Eckersley, Alan Flores-López, J. Alex Halderman, Jacob Hoffman-Andrews, James Kasten, Eric Rescorla, Seth Schoen, and Brad Warren. "Let's Encrypt: An Automated Certificate Authority to Encrypt the Entire Web". In: ACM Conference on Computer and Communications Security (CCS), 2019.

[2] Akamai. Whitepaper: DNS Reflection, Amplification, \& DNS Water-torture. Accessed: 2021. URL: https ://www. akamai .com/uk/en/multimedia/documents / technical-publication/dns-reflection-vs-dns-mirai-technical-publication.pdf.

[3] Furkan Alaca, AbdelRahman Abdou, and P.C. van Oorschot. "Comparative Analysis and Framework Evaluating Mimicry-Resistant and Invisible Web Authentication Schemes”. In: IEEE Trans. Dependable and Secure Computing (TDSC) (2021), pp. 534-539.

[4] Marios Anagnostopoulos, Georgios Kambourakis, Elisavet Konstantinou, and Stefanos Gritzalis. "DNSSEC vs. DNSCurve: A side-by-side comparison”. In: Situational Awareness in Computer Network Defense: Principles, Methods and Applications. IGI Global, 2012, pp. 201-220.

[5] Marios Anagnostopoulos, Georgios Kambourakis, Panagiotis Kopanos, Georgios Louloudakis, and Stefanos Gritzalis. "DNS Amplification Attack Revisited". In: Computers \& Security 39 (2013), pp. 475-485. 
[6] Manos Antonakakis, Tim April, Michael Bailey, Matt Bernhard, Elie Bursztein, Jaime Cochran, Zakir Durumeric, J. Alex Halderman, Luca Invernizzi, Michalis Kallitsis, Deepak Kumar, Chaz Lever, Zane Ma, Joshua Mason, Damian Menscher, Chad Seaman, Nick Sullivan, Kurt Thomas, and Yi Zhou. "Understanding the Mirai Botnet”. In: USENIX Security Symposium. 2017.

[7] Noah Apthorpe, Dillon Reisman, and Nick Feamster. "Closing the blinds: Four strategies for protecting smart home privacy from network observers". In: arXiv preprint arXiv:1705.06809 (2017).

[8] Roy Arends, Scott Rose, Matt Larson, Dan Massey, and Rob Austein. DNS Security Introduction and Requirements. RFC 4033. Mar. 2005. URL: https://tools.ietf.org/ $\mathrm{html} / \mathrm{rfc} 4033$.

[9] Roy Arends, Scott Rose, Matt Larson, Dan Massey, and Rob Austein. Protocol Modifications for the DNS Security Extensions. RFC 4035. Mar. 2005. URL: https://tools. ietf.org/html/rfc4035.

[10] Roy Arends, Scott Rose, Matt Larson, Dan Massey, and Rob Austein. Resource Records for the DNS Security Extensions. RFC 4034. Mar. 2005. URL: https://tools. ietf.org/html/rfc4034.

[11] Simurgh Aryan, Homa Aryan, and J Alex Halderman. "Internet censorship in Iran: A first look". In: USENIX Workshop on Free and Open Communications on the Internet (FOCI). 2013.

[12] Elaine Barker. Recommendation for Key Management-Special Publication 800-57 Part 1. National Institute of Standards and Technology (NIST). 2020.

[13] Richard Barnes, Jacob Hoffman-Andrews, Daniel McCarney, and James Kasten. Automatic Certificate Management Environment (ACME). RFC 8555. 2019. URL: https://rfc-editor.org/rfc/rfc8555.txt. 
[14] Daniel J Bernstein. “Cryptography in NaCl”. In: (2009).

[15] Daniel J Bernstein. DNSCurve: Usable security for DNS. 2009. uRL: https://dnscurve. org.

[16] Sarah Bird, Ilana Segall, and Martin Lopatka. "Replication: Why We Still Can't Browse in Peace: On the Uniqueness and Reidentifiability of Web Browsing Histories”. In: USENIX Symposium on Usable Privacy and Security (SOUPS). 2020.

[17] David Bisson. Self-Signed Certificates: Cyber-criminals Are Turning This Strength into a Vulnerability. 2019. URL: https : // www . venafi .com/blog / self - signed certificates-cyber-criminals-are-turning-strength-into-a-vulnerability.

[18] Sharon Boeyen, Stefan Santesson, Tim Polk, Russ Housley, Stephen Farrell, and Dave Cooper. Internet X.509 Public Key Infrastructure Certificate and Certificate Revocation List (CRL) Profile. RFC 5280. 2008. uRL: https://rfc-editor.org/rfc/ rfc5280.txt.

[19] Stéphane Bortzmeyer. DNS Privacy Considerations. RFC 7626. Aug. 2015. URL: https://rfc-editor.org/rfc/rfc7626.txt.

[20] Stéphane Bortzmeyer. DNS Query Name Minimisation to Improve Privacy. RFC 7816. Mar. 2016. URL: https://tools.ietf.org/html/rfc7816.

[21] Jonas Bushart and Christian Rossow. "Padding Ain't Enough: Assessing the Privacy Guarantees of Encrypted DNS". In: USENIX Workshop on Free and Open Communications on the Internet (FOCI). 2020.

[22] Patricia Callejo, Rubén Cuevas, Narseo Vallina-Rodriguez, and Ángel Cuevas Rumin. "Measuring the global recursive DNS infrastructure: a view from the edge". In: IEEE Access 7 (2019), pp. 168020-168028. 
[23] Laurent Chuat, AbdelRahman Abdou, Ralf Sasse, Christoph Sprenger, David Basin, and Adrian Perrig. "SoK: Delegation and Revocation, the Missing Links in the Web's Chain of Trust”. In: IEEE European Symposium on Security and Privacy (EuroSP). 2020.

[24] Taejoong Chung, Yabing Liu, David Choffnes, Dave Levin, Bruce MacDowell Maggs, Alan Mislove, and Christo Wilson. "Measuring and applying invalid SSL certificates: The silent majority". In: ACM Internet Measurement Conference (IMC). 2016.

[25] Taejoong Chung, Roland van Rijswijk-Deij, Balakrishnan Chandrasekaran, David Choffnes, Dave Levin, Bruce M Maggs, Alan Mislove, and Christo Wilson. “A Longitudinal, End-to-End View of the DNSSEC Ecosystem”. In: USENIX Security Symposium. 2017.

[26] Carlo Contavalli, Wilmer van der Gaast, David C. Lawrence, and Warren "Ace" Kumari. Client Subnet in DNS Queries. RFC 7871. 2016. URL: https://rfc-editor. org/rfc/rfc7871.txt.

[27] Alex Cowperthwaite and Anil Somayaji. "The futility of DNSSEC". In: Annual Symposium Information Assurance (ASIA). 2010.

[28] Tianxiang Dai, Haya Shulman, and Michael Waidner. "DNSSEC misconfigurations in popular domains". In: Conference on Cryptology and Network Security (CANS). Springer. 2016.

[29] Matthew Dempsky. DNSCurve: Link-Level Security for the Domain Name System. Internet-Draft. Internet Engineering Task Force, Feb. 2010.

[30] Frank Denis. Anonymized DNSCrypt specification. 2019. uRL: https://github.com/ DNSCrypt/dnscrypt-protocol/blob/master/ANONYMIZED-DNSCRYPT.txt. 
[31] Frank Denis. DNSCrypt version 2 protocol specification. 2017. URL: https://github. com/DNSCrypt/dnscrypt-protocol/blob/master/DNSCRYPT-V2-PROTOCOL. txt.

[32] Sara Dickinson. DNS Privacy Public Resolvers. 2019. uRL: https://dnsprivacy.org/ wiki/display/DP/DNS+Privacy+Public+Resolvers.

[33] Digicert. Moving forward: What DigiCert's CT2 log retirement means for you. 2020. URL: https://www.digicert.com/dc/blog/digicert-statement-on-ct2-log/.

[34] Roger Dingledine, Nick Mathewson, and Paul Syverson. Tor: The second-generation onion router. Tech. rep. Naval Research Lab Washington DC, 2004.

[35] Zakir Durumeric, David Adrian, Ariana Mirian, Michael Bailey, and J Alex Halderman. "A search engine backed by Internet-wide scanning". In: ACM Conference on Computer and Communications Security (CCS). 2015.

[36] Zakir Durumeric, James Kasten, Michael Bailey, and J. Alex Halderman. "Analysis of the HTTPS Certificate Ecosystem". In: ACM Conference on Internet Measurement Conference (IMC). 2013.

[37] Zakir Durumeric, Eric Wustrow, and J Alex Halderman. "ZMap: Fast Internet-wide scanning and its security applications". In: USENIX Security Symposium. 2013.

[38] CA/Browser Forum. Baseline Requirements for the Issuance and Management of Publicly-Trusted Certificates, v.1.2.3. 2014.

[39] GoDaddy. Renewing my SSL Certificate. URL: https :// ca . godaddy.com/help/ renewing-my-ssl-certificate-864.

[40] Fernando Gont. Introduction to DNS Privacy. 2019. uRL: https://www.internetsociety. org/resources/deploy360/dns-privacy/intro/. 
[41] Benjamin Greschbach, Tobias Pulls, Laura M. Roberts, Philipp Winter, and Nick Feamster. "The Effect of DNS on Tor's Anonymity”. In: CoRR abs/1609.08187 (2016).

[42] C. Grothoff, M. Wachs, and M. Ermert. "NSA's MORECOWBELL: Knell for DNS". 2017. URL: http://cryptome.org/2015/01/MORECOWBELL-Analysis-Grothoffetal.pdf.

[43] John A. Hawkinson and Tony J. Bates. Guidelines for creation, selection, and registration of an Autonomous System (AS). RFC 1930. Mar. 1996. URL: https://rfceditor.org/rfc/rfc1930.txt.

[44] Nguyen Phong Hoang, Sadie Doreen, and Michalis Polychronakis. "Measuring I2P Censorship at a Global Scale”. In: USENIX Workshop on Free and Open Communications on the Internet (FOCI). 2019.

[45] Nguyen Phong Hoang, Ivan Lin, Seyedhamed Ghavamnia, and Michalis Polychronakis. "K-resolver: Towards Decentralizing Encrypted DNS Resolution”. In: arXiv preprint arXiv:2001.08901 (2020).

[46] Paul E. Hoffman and Patrick McManus. DNS Queries over HTTPS (DoH). RFC 8484. Oct. 2018. URL: https://rfc-editor.org/rfc/rfc8484.txt.

[47] Austin Hounsel, Kevin Borgolte, Paul Schmitt, and Nick Feamster. "D-DNS: Towards Re-Decentralizing the DNS”. In: arXiv preprint arXiv:2002.09055 (2020).

[48] Rebekah Houser, Zhou Li, Chase Cotton, and Haining Wang. "An Investigation on Information Leakage of DNS over TLS”. In: ACM International Conference on Emerging Networking Experiments And Technologies (CoNEXT). 2019.

[49] Z. Hu, L. Zhu, J. Heidemann, A. Mankin, D. Wessels, and P. Hoffman. Specification for DNS over Transport Layer Security (TLS). RFC 7858. RFC Editor, May 2016. URL: https://tools.ietf.org/html/rfc7858. 
[50] Bert Hubert and Remco Mook. Measures for Making DNS More Resilient against Forged Answers. RFC 5452. Jan. 2009. uRL: https://rfc-editor.org/rfc/rfc5452.txt.

[51] Christian Huitema, Melinda Shore, Allison Mankin, Sara Dickinson, and Janardhan Iyengar. Specification of DNS over Dedicated QUIC Connections. Sept. 2019. URL: https://tools.ietf.org/html/draft-huitema-quic-dnsoquic-07.

[52] George Hurlburt. "The Internet of Things... of All Things". In: ACM XRDS:Crossroads 22.2 (2016), 22-26.

[53] Geoff Huston. DNSSEC Validation Revisited. 2020. uRL: https://blog.apnic.net/ 2020/03/02/dnssec-validation-revisited/.

[54] Jana Iyengar and Martin Thomson. QUIC: A UDP-Based Multiplexed and Secure Transport. Internet-Draft draft-ietf-quic-transport-32. Work in Progress. Internet Engineering Task Force, Oct. 2020. 200 pp. URL: https://datatracker.ietf.org/doc/ $\mathrm{html} /$ draft-ietf-quic-transport-32.

[55] Philipp Jeitner, Haya Shulman, and Michael Waidner. "The Impact of DNS Insecurity on Time". In: Annual IEEE/IFIP International Conference on Dependable Systems and Networks (DSN). 2020.

[56] Dan Kaminsky. Black ops 2008: Its the end of the cache as we know it. Black Hat USA, 2008.

[57] Florian Kammüller, Yoney Kirsal-Ever, and Xiaochun Cheng. "DNSSEC in isabellereplay attack and origin authentication". In: IEEE International Conference on Systems, Man, and Cybernetics. 2013.

[58] Aminollah Khormali, Jeman Park, Hisham Alasmary, Afsah Anwar, Muhammad Saad, and David Mohaisen. "Domain name system security and privacy: A contemporary survey". In: Computer Networks (2020), p. 107699. 
[59] Tae Hyun Kim and Douglas Reeves. "A survey of domain name system vulnerabilities and attacks". In: Journal of Surveillance, Security and Safety (JSSS) 1.1 (2020), pp. 34-60.

[60] Eric Kinnear, Patrick McManus, Tommy Pauly, and Christopher A. Wood. Oblivious DNS Over HTTPS. Internet-Draft draft-pauly-dprive-oblivious-doh-03. Work in Progress. Dec. 2020. 18 pp. URL: https://datatracker.ietf.org/doc/html/draftpauly-dprive-oblivious-doh-03.

[61] Ben Laurie, Adam Langley, and Emilia Kasper. Certificate Transparency. RFC 6962. 2013. URL: https://rfc-editor.org/rfc/rfc6962.txt.

[62] Neal Leavitt. "Internet security under attack: The undermining of digital certificates". In: Computer 44.12 (2011), pp. 17-20.

[63] Chaoyi Lu, Baojun Liu, Zhou Li, Shuang Hao, Haixin Duan, Mingming Zhang, Chunying Leng, Ying Liu, Zaifeng Zhang, and Jianping Wu. "An End-to-End, LargeScale Measurement of DNS-over-Encryption: How Far Have We Come?" In: ACM Internet Measurement Conference (IMC). 2019.

[64] Robert Lychev, Samuel Jero, Alexandra Boldyreva, and Cristina Nita-Rotaru. "How secure and quick is QUIC? Provable security and performance analyses". In: IEEE Symposium on Security and Privacy (S\&P). 2015.

[65] Tasnuva Mahjabin and Yang Xiao. "Mitigation process for DNS flood attacks". In: IEEE Annual Consumer Communications \& Networking Conference (CCNC). 2019.

[66] Keyu Man, Zhiyun Qian, Zhongjie Wang, Xiaofeng Zheng, Youjun Huang, and Haixin Duan. "DNS Cache Poisoning Attack Reloaded: Revolutions with Side Channels". In: ACM Conference on Computer and Communications Security (CCS). 2020. 
[67] Keith Martin. Everyday Cryptography: Fundamental Principles \& Applications. 2nd. Oxford University Press, June 2017.

[68] Alexander Mayrhofer. The EDNS(0) Padding Option. RFC 7830. May 2016. uRL: https://rfc-editor.org/rfc/rfc7830.txt.

[69] P. Mockapetris. Domain Names - Implementation and Specification. RFC 1035. RFC Editor, Nov. 1987. uRL: https://tools.ietf.org/html/rfc1035.

[70] Paul Mockapetris. Domain Names - Concepts and Facilities. RFC 1034. Nov. 1987. URL: https://tools.ietf.org/html/rfc1034.

[71] Giovane C. M. Moura, Sebastian Castro, Wes Hardaker, Maarten Wullink, and Cristian Hesselman. "Clouding up the Internet: How Centralized is DNS Traffic Becoming?" In: ACM Internet Measurement Conference (IMC). 2020.

[72] Alec Muffett. "No Port 53, Who Dis? A Year of DNS over HTTPS over Tor". In: NDSS DNS Privacy Workshop, 2021.

[73] Zubair Nabi. "The Anatomy of Web Censorship in Pakistan". In: USENIX Workshop on Free and Open Communications on the Internet (FOCI). 2013.

[74] Arian Akhavan Niaki, Shinyoung Cho, Zachary Weinberg, Nguyen Phong Hoang, Abbas Razaghpanah, Nicolas Christin, and Phillipa Gill. "IClab: a global, longitudinal internet censorship measurement platform". In: IEEE Symposium on Security and Privacy $(S \& P) .2020$.

[75] Yoav Nir and Adam Langley. ChaCha20 and Poly1305 for IETF Protocols. RFC 8439. June 2018. uRL: https://rfc-editor.org/rfc/rfc8439.txt.

[76] Gunter Ollmann. "The Pharming Guide: Understanding \& Mitigating DNS-related Attacks by Phishers". In: 2005. uRL: https://research.nccgroup.com/wp-content/ uploads/2020/07/thepharmingguide.pdf. 
[77] Paul C. van Oorschot. Computer Security and the Internet: Tools and Jewels. Springer Nature, 2020.

[78] Liran Orevi, Amir Herzberg, and Haim Zlatokrilov. "DNS-DNS: DNS-based denat scheme". In: International Conference on Cryptology and Network Security. Springer. 2018, pp. 69-88.

[79] Michal Špaček. Maximum HTTPS certificate lifetime to be 1 year soon. 2020. URL: https://www.michalspacek.com/maximum-https-certificate-lifetime-to-be-1-yearsoon.

[80] Paul Pearce, Ben Jones, Frank Li, Roya Ensafi, Nick Feamster, Nick Weaver, and Vern Paxson. “Global Measurement of DNS Manipulation”. In: USENIX Security Symposium. 2017.

[81] The Tor Project. Reporting Bad Relays. 2018. uRL: https :// trac.torproject.org/ projects/tor/wiki/doc/ReportingBadRelays.

[82] Venugopalan Ramasubramanian and Emin Gün Sirer. "Perils of transitive trust in the domain name system”. In: ACM Internet Measurement Conference (IMC). 2005.

[83] Audrey Randall, Enze Liu, Gautam Akiwate, Ramakrishna Padmanabhan, Geoffrey M. Voelker, Stefan Savage, and Aaron Schulman. "Trufflehunter: Cache Snooping Rare Domains at Large Public DNS Resolvers". In: ACM Internet Measurement Conference (IMC). 2020.

[84] Rapid7. Project Sonar. URL: https://opendata.rapid7.com/about/.

[85] Tirumaleswar Reddy, Dan Wing, and Prashanth Patil. DNS over Datagram Transport Layer Security (DTLS). RFC 8094. Feb. 2017. uRL: https://tools.ietf.org/html/ rfc8094.

[86] Eric Rescorla and Nagendra Modadugu. Datagram Transport Layer Security Version 1.2. RFC 6347. 2012. URL: https://rfc-editor.org/rfc/rfc6347.txt. 
[87] Thijs Rozekrans, Matthijs Mekking, and Javy de Koning. “Defending against DNS reflection amplification attacks". In: University of Amsterdam System \& Network Engineering RPl (2013).

[88] Ali Sadeghi Jahromi and AbdelRahman Abdou. "Comparative Analysis of DoT and HTTPS Certificate Ecosystems”. In: NDSS Workshop on Measurements, Attacks, and Defenses for the Web (MADWeb). 2021.

[89] Mahrud Sayrafi. Introducing DNS Resolver for Tor. 2018. uRL: https://blog.cloudflare. com/welcome-hidden-resolver/.

[90] Quirin Scheitle, Oliver Gasser, Theodor Nolte, Johanna Amann, Lexi Brent, Georg Carle, Ralph Holz, Thomas C Schmidt, and Matthias Wählisch. “The rise of certificate transparency and its implications on the internet ecosystem". In: ACM Internet Measurement Conference (IMC). 2018.

[91] Paul Schmitt, Anne Edmundson, Allison Mankin, and Nick Feamster. "Oblivious DNS: Practical privacy for DNS queries". In: Privacy Enhancing Technologies (PETS) (2019).

[92] Haya Shulman. "Pretty Bad Privacy: Pitfalls of DNS Encryption". In: Workshop on Privacy in the Electronic Society (WPES). 2014.

[93] Sandra Siby, Marc Juárez, Claudia Díaz, Narseo Vallina-Rodriguez, and Carmela Troncoso. "Encrypted DNS -> Privacy? A Traffic Analysis Perspective". In: CoRR abs/1906.09682 (2019). URL: http://arxiv.org/abs/1906.09682.

[94] Sudheesh Singanamalla, Suphanat Chunhapanya, Marek Vavruša, Tanya Verma, Peter Wu, Marwan Fayed, Kurtis Heimerl, Nick Sullivan, and Christopher Wood. Oblivious DNS over HTTPS (ODoH): A Practical Privacy Enhancement to DNS. 2020. arXiv: 2011.10121 [Cs.CR]. 
[95] Kushagra Singh, Gurshabad Grover, and Varun Bansal. "How India Censors the Web”. In: ACM Conference on Web Science (WebSci). 2020.

[96] Sooel Son and Vitaly Shmatikov. “The hitchhiker's guide to DNS cache poisoning”. In: International Conference on Security and Privacy in Communication Systems. Springer. 2010, pp. 466-483.

[97] Emily Stark, Ryan Sleevi, Rijad Muminovic, Devon O’Brien, Eran Messeri, Adrienne Porter Felt, Brendan McMillion, and Parisa Tabriz. "Does certificate transparency break the web? Measuring adoption and error rate". In: IEEE Symposium on Security and Privacy (S\&P). 2019.

[98] Pouyan Fotouhi Tehrani, Eric Osterweil, Jochen H Schiller, Thomas C Schmidt, and Matthias Wählisch. "Who ya gonna call? (Alerting Authorities): Measuring Namespaces, Web Certificates, and DNSSEC'. In: arXiv preprint arXiv:2008.10497 (2020).

[99] Kutub Thakur, Md Liakat Ali, Sandra Kopecky, Abu Kamruzzaman, and Lixin Tao. “Connectivity, Traffic Flow and Applied Statistics in Cyber Security”. In: IEEE International Conference on Smart Cloud (SmartCloud). 2016.

[100] Emin Topalovic, Brennan Saeta, Lin-Shung Huang, Collin Jackson, and Dan Boneh. “Towards short-lived certificates”. In: Web 2.0 Security and Privacy (2012).

[101] Sadegh Torabi, Amine Boukhtouta, Chadi Assi, and Mourad Debbabi. "Detecting Internet abuse by analyzing passive DNS traffic: A survey of implemented systems". In: IEEE Communications Surveys \& Tutorials (COMST) (2018), pp. 3389-3415.

[102] Nevena Vratonjic, Julien Freudiger, Vincent Bindschaedler, and Jean-Pierre Hubaux. “The Inconvenient Truth About Web Certificates". In: Economics of Information Security and Privacy III. Ed. by Bruce Schneier. 2013, pp. 79-117.

[103] Wouter Wijngaards and Glen Wiley. Confidential DNS. 2015. uRL: https://tools. ietf.org/html/draft-wijngaards-dnsop-confidentialdns-03. 
[104] Philipp Winter, Richard Köwer, Martin Mulazzani, Markus Huber, Sebastian Schrittwieser, Stefan Lindskog, and Edgar Weippl. "Spoiled Onions: Exposing Malicious Tor Exit Relays". In: Privacy Enhancing Technologies. Springer International Publishing, 2014, pp. 304-331.

[105] Philipp Winter and Stefan Lindskog. "How the Great Firewall of China is Blocking Tor". In: USENIX Workshop on Free and Open Communications on the Internet (FOCI). 2012.

[106] Tarun Kumar Yadav, Akshat Sinha, Devashish Gosain, Piyush Kumar Sharma, and Sambuddho Chakravarty. "Where The Light Gets In: Analyzing Web Censorship Mechanisms in India”. In: ACM Internet Measurement Conference (IMC). 2018.

[107] He Yan, Eric Osterweil, Jon Hajdu, Jonas Acres, and Dan Massey. "Limiting replay vulnerabilities in DNSSEC". In: IEEE Workshop on Secure Network Protocols. 2008.

[108] Shui Yu. Distributed denial of service attack and defense. Springer, 2014.

[109] Liang Zhu, Zi Hu, John Heidemann, Duane Wessels, Allison Mankin, and Nikita Somaiya. "Connection-oriented DNS to improve privacy and security". In: IEEE Symposium on Security and Privacy (S\&P). 2015.

[110] Futai Zou, Siyu Zhang, Bei Pei, Li Pan, Linsen Li, and Jianhua Li. "Survey on domain name system security". In: IEEE First International Conference on Data Science in Cyberspace (DSC). 2016. 